\title{
Synthesis, spectral correlations and antimicrobial activities of some 2-hydroxyphenyl-styrylketone
}

\author{
K. Sathiyamoorthi ${ }^{1}$, V. Mala ${ }^{1}$, R. Suresh ${ }^{1}$, S. P. Sakthinathan ${ }^{1}$, \\ D. Kamalakkannan ${ }^{1}$, K. Ranganathan ${ }^{1}$, R. Arulkumaran ${ }^{1}$, R. Sundararajan ${ }^{1}$, \\ S. Vijayakumar ${ }^{1}$, G. Vanangamudi ${ }^{1, *}$, G. Thirunarayanan ${ }^{2}$ \\ ${ }^{1}$ PG \& Research Department of Chemistry, Government Arts College C-Mutlur, \\ Chidambaram - 608102, India. \\ ${ }^{2}$ Department of Chemistry, Annamalai University, Annamalainagar - 608002, India \\ *E-mail address: drgvsibi@gmail.com
}

\begin{abstract}
Some 2'-hydroxyphenylchalcones have been synthesized under microwave irradiation by ClaisenSchmidt condensation between substituted 2-hydroxyacetophenone and substituted benzaldehydes using catalytic amount of $\mathrm{SiO}_{2}-\mathrm{H}_{3} \mathrm{PO}_{4}$. These chalcones were established by their physical constants and spectroscopic data published earlier. The UV, IR, ${ }^{1} \mathrm{H}$ NMR and ${ }^{13} \mathrm{C}$ NMR spectral data of these chalcone have correlated with Hammett substituent constants, F and R parameters. All the compounds have been subjected to screened for antimicrobial activity.
\end{abstract}

Keywords: Solvent-free synthesis; $\mathrm{SiO}_{2}-\mathrm{H}_{3} \mathrm{PO}_{4} ; 2$ '-hydroxyphenylchalcones; UV; IR; NMR spectra

\section{INTRODUCTION}

Chalcones (1,3-diarylpropinones) are the condensation product of aryl ketones and aryl aldehydes and they consisting of two aryl rings linked by ketone and unsaturated moieties. Chalcones have been synthesised [1] natural products extracted from plants [2] belonging to the flavonoid family [3-5]. The compounds with the backbone [6] of chalcone have been reported to exhibit a wide variety of pharmacological effects including, antimalarial [7-9], antiviral [10-12], antibacterial [13-15], antiplosmodial [16], antifungal [17-19], anticancer [20-22], anti-leishmaninal [23], anti-inflammatory [24], antipyretic [25], analgesic [26], antiulcerative [27], antihyperglycemic [28], antioxidant [29], antiinvasive [30], antiplatelet [31] and insect antifeedent [32], antihyperglycemic [33], antifeedent [34] immunomodulatory [35], inhibition of chemical mediators release [36], inhibition of leukotriene $\mathrm{B}_{4}$ [37], inhibition of tyrosinase [38], and inhibition of aldose reductase [39] activities. At present, scientists have paid more interest to correlate the group frequencies of spectral data with Hammett substituent constants to explain the substituent effects of organic compounds, Subramanian et al. [40] have studied the effect of substituents through spectral linearity and antimicrobial activities of some furyl chalcones. In their studies, they observed satisfactory and good correlations for each spectral data with Hammett substituent constants, F and $\mathrm{R}$ parameters. In the present investigation, the authors have synthesized a series of some 2'hydroxychalcones, studied the spectral correlations with Hammett substituent constants, F and R parameters using single and multi-linear regression analysis and evaluated their antibacterial antifungal activities [47-49]. 


\section{EXPERIMENTAL}

\section{1. Materials and Methods}

All the chemicals involved in the present investigation, have been procured from SigmaAldrich and E-Merck chemical companies. Melting points of all imines have been determined in open glass capillaries on SUNTEX melting point apparatus and are uncorrected. The UV spectra of all the imines, synthesized, have been recorded with ELICO-BL222 spectrophotometer $\left(\lambda_{\max } \mathrm{nm}\right)$ in spectral grade methanol solvent. Infrared spectra $\left(\mathrm{KBr}, 4000-400 \mathrm{~cm}^{-1}\right)$ have been recorded on AVATAR-300 Fourier transform spectrophotometer. The NMR spectra were recorded in Bruker AV400 NMR spectrometer operating at $400 \mathrm{MHz}$ has been utilized for recording ${ }^{1} \mathrm{H}$ NMR spectra and $100 \mathrm{MHz}$ for ${ }^{13} \mathrm{C}$ spectra in $\mathrm{CDCl}_{3}$ solvent using TMS as internal standard.

\section{2. Preparation of chalcones}

An appropriate equi-molar quantities of 2-hydroxyacetophenone (2 mmol), substituted benzaldehydes $(2 \mathrm{mmol})$ and $\mathrm{SiO}_{2}-\mathrm{H}_{3} \mathrm{PO}_{4}$ [41] $(0.5 \mathrm{~g})$ have been taken in borosil tube and tightly capped. The mixture has been subjected to microwave irradiation for 6-12 minutes in a microwave oven (Scheme 1) (Samsung, Microwave Oven, and 100-700 W) and then cooled to room temperature. After separating the organic layer with dichloromethane the solid product has been obtained on evaporation.

The solid, on recrystallization with benzene-hexane mixture gives glittering product. The insoluble catalyst has been recycled by washing with ethyl acetate $(8 \mathrm{~mL})$ followed by drying in an oven at $100{ }^{\circ} \mathrm{C}$ for $1 \mathrm{~h}$ and reused for further reactions. The analytical data of chalcones are presented in Table 1.<smiles>CC(=O)c1ccccc1O</smiles><smiles>[X]c1ccc(C=O)cc1</smiles><smiles>[X]c1ccc(C=CC(=O)c2ccccc2O)cc1</smiles>

$\mathrm{X}=\mathrm{H}, 2-\mathrm{Br}, 3-\mathrm{Cl}, 4-\mathrm{Cl}, 2-\mathrm{F}, 4-\mathrm{F}, 2-\mathrm{OCH}_{3}, 4-\mathrm{OCH}_{3}, 4-\mathrm{NO}_{2}$

Scheme 1. Synthesis of substituted 2-hydroxyphenyl-styrylketone.

Table 1. Anaytical and physical constant of 2'-hydroxyphenylchalcones.

\begin{tabular}{|c|c|c|c|c|}
\hline Entry & $\mathbf{X}$ & $\mathbf{M .} \mathbf{F}$. & $\mathbf{M} . \mathbf{W}$. & $\mathbf{m . p .}\left({ }^{\circ} \mathbf{C}\right)$ \\
\hline 1 & $\mathrm{H}$ & $\mathrm{C}_{15} \mathrm{H}_{12} \mathrm{O}_{2}$ & 224 & $90-91(89)[42]$ \\
\hline 2 & $2-\mathrm{Br}$ & $\mathrm{C}_{15} \mathrm{H}_{11} \mathrm{BrO}_{2}$ & 303 & $113-114(112-113)[38]$ \\
\hline 3 & $3-\mathrm{Cl}$ & $\mathrm{C}_{15} \mathrm{H}_{11} \mathrm{ClO}_{2}$ & 258 & $63-64(60-62)[43]$ \\
\hline 4 & $4-\mathrm{Cl}$ & $\mathrm{C}_{15} \mathrm{H}_{11} \mathrm{ClO}_{2}$ & 258 & $152(151-152)[42]$ \\
\hline 5 & $2-\mathrm{F}$ & $\mathrm{C}_{15} \mathrm{H}_{11} \mathrm{FO}_{2}$ & 242 & $59(56-58)[43]$ \\
\hline
\end{tabular}




\begin{tabular}{|c|c|c|c|c|}
\hline 6 & $4-\mathrm{F}$ & $\mathrm{C}_{15} \mathrm{H}_{11} \mathrm{FO} 2$ & 242 & $50(46-48)[43]$ \\
\hline 7 & $2-\mathrm{OCH}_{3}$ & $\mathrm{C}_{16} \mathrm{H}_{14} \mathrm{O}_{3}$ & 254 & $122-123(120-122)[43]$ \\
\hline 8 & $4-\mathrm{OCH}_{3}$ & $\mathrm{C}_{16} \mathrm{H}_{14} \mathrm{O}_{3}$ & 254 & $65(68-70)[42]$ \\
\hline 9 & $4-\mathrm{NO}_{2}$ & $\mathrm{C}_{15} \mathrm{H}_{11} \mathrm{NO}_{4}$ & 269 & $61-62(58-60)[43]$ \\
\hline
\end{tabular}

\section{RESULTS AND DISCUSSION}

\section{1. Spectral linearity}

In the present study the Hammett spectral linearity of these synthesised chalcones has been studied by evaluating the substituent effects $[1,16,40,41]$ on the group frequencies. The assigned spectroscopic data of all chalcones such as absorption maximum $\lambda_{\max }(\mathrm{nm})$ of carbonyl groups, infrared carbonyl stretches of $v \mathrm{CO}$-cis and s-trans, the deformation modes of vinyl part $\mathrm{CH}$ out of plane, in-plane, $\mathrm{CH}=\mathrm{CH}$ and $>\mathrm{C}=\mathrm{C}<$ out of planes $\left(\mathrm{cm}^{-1}\right)$, NMR chemical shifts $\delta(\mathrm{ppm})$ of $\mathrm{H}_{\alpha}, \mathrm{H}_{\beta}$, $\mathrm{C}_{\alpha}, \mathrm{C}_{\beta}, \mathrm{CO}$ are assigned and these data are correlated with various substituent constants.

\section{1. 1. UV-spectral study}

The absorption maxima $\left(\lambda_{\max } \mathrm{nm}\right)$ of these chalcones were assigned and presented in Table 2. These absorption maxima $\left(\lambda_{\max } \mathrm{nm}\right)$ of these chalcones were correlated with Hammett substituent constants and $\mathrm{F}$ and $\mathrm{R}$ parameters using single and multi-linear regression analysis [1,16,40,41]. Hammett correlation involving the group frequencies and absorption maxima, the form of the Hammett equation employed is

$$
\lambda=\rho \sigma+\lambda_{\mathrm{o}}
$$

where $\lambda_{\mathrm{o}}$ is the frequency for the parent member of the series.

The results of statistical analysis [1, 16, 40, 41] of these values with Hammett substituent constants $\sigma(r=0.902), \sigma^{+}(r=0.903)$ has satisfactory correlation, $F$ and $R$ parameters are presented in Table 3. The Hammett $\sigma_{\mathrm{I}}, \sigma_{\mathrm{R}}$, constants and $\mathrm{F}$ and $\mathrm{R}$ parameters gave poor correlation. This is due to the polar, filed, resonance and inductive effects of the substituents were weak for predicting the reactivity on the absorption. This is evident with resonance conjugative structure shown in Figure 1.

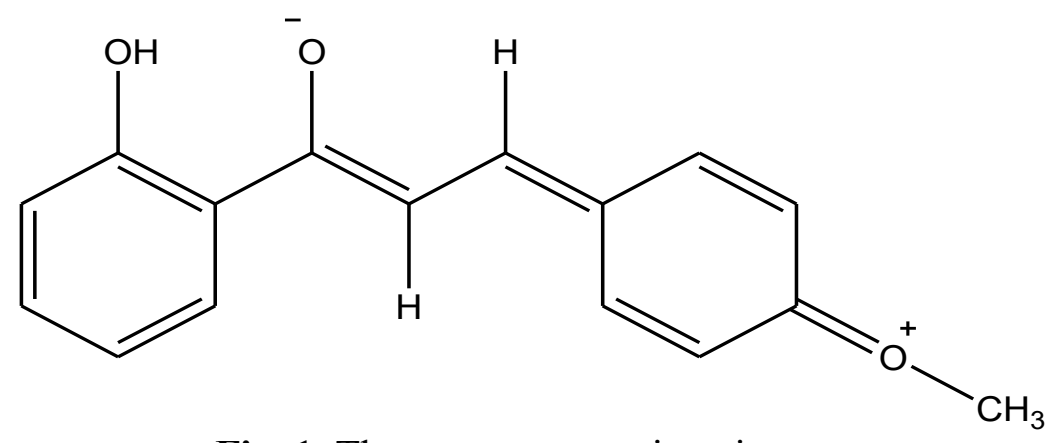

Fig. 1. The resonance-conjugative structure 
The multi regression analysis of these frequencies of all ketones with inductive, resonance and Swain - Lupton's [44] constants produce satisfactory correlations as evident in equations (2 and $3)$.

$$
\begin{gathered}
\lambda_{\max }(\mathrm{nm})=\begin{array}{c}
313.393( \pm 20.615)+16.822( \pm 41.912) \sigma_{\mathrm{I}}-28.932( \pm 32.485) \sigma_{\mathrm{R}} \\
(R=0.936, \quad \mathrm{n}=9, \quad \mathrm{P}>90 \%)
\end{array} \\
\lambda_{\max }(\mathrm{nm})=\begin{array}{c}
312.445( \pm 16.516)+15.584( \pm 32.921) \mathrm{F}-27.743( \pm 28.467) \mathrm{R} \\
(R=0.945, \quad \mathrm{n}=9, \quad \mathrm{P}>90 \%)
\end{array}
\end{gathered}
$$

\section{1.2. IR spectral study}

The assigned carbonyl stretching frequencies $\left(\mathrm{cm}^{-1}\right)$ of $s$-cis and s-trans isomers of present study are presented in Table 2 and the corresponding conformers are shown in Fig. 2. The stretching frequencies for carbonyl absorption are assigned based on the assignments made by Hays and Timmons [45] for s-cis and s-trans conformers at 1690 and $1670 \mathrm{~cm}^{-1}$, respectively.

These data have been correlated with Hammett substituent constants and Swain-Lupton constants[44] and are presented in Table 3. In this correlation the structure parameter Hammett equation employed is as shown in the following equation:

$$
v=\rho \sigma+v_{0}
$$

Where $v$ is the carbonyl frequencies of substituted system and $v_{0}$ is the corresponding quantity of unsubstitued system; $\sigma$ is a Hammett substituent constant, which in principle is characteristics of the substituent and $\rho$ is a reaction constant which is depend upon the nature of the reaction. 
Table 2. The ultraviolet absorption maxima $(\lambda \max , \mathrm{nm})$, infrared absorptions $\left(\mathrm{v}, \mathrm{cm}^{-1}\right)$ and NMR chemical shifts $(\delta, \mathrm{ppm})$ of substituted 2-hydroxyphenyl-styrylketone.

\begin{tabular}{|c|c|c|c|c|c|c|c|c|c|c|c|c|c|}
\hline 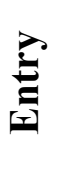 & $x$ & ב气 & రీ & & $\stackrel{\vec{\theta}}{\overrightarrow{0}}$ & $\underbrace{8}_{0}$ & 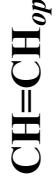 & $\bigcup_{0}^{\tilde{\theta}}$ & $\mathbb{I}^{8}$ & 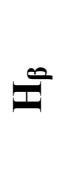 & $ن^{8}$ & $u^{\infty}$ & O \\
\hline 1 & $I$ & $\begin{array}{l}\hat{a} \\
\hat{n}\end{array}$ & $\frac{\underset{f}{f}}{\underset{J}{\sigma}}$ & $\begin{array}{l}\text { 尺) } \\
\stackrel{0}{0}\end{array}$ & $\begin{array}{l}8 \\
\Xi \\
\text { 三 }\end{array}$ & 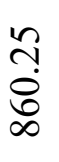 & $\begin{array}{l}\text { ণิ } \\
\stackrel{+}{ \pm} \\
\text { O }\end{array}$ & 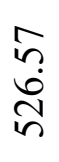 & $\underset{\sim}{\stackrel{J}{*}}$ & $\hat{\sigma}$ & 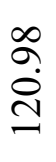 & $\begin{array}{l}\infty \\
\stackrel{\infty}{\infty} \\
\stackrel{0}{n}\end{array}$ & $\begin{array}{l}\circ \\
\circ \\
\sigma\end{array}$ \\
\hline 2 & $\stackrel{\vec{m}}{\grave{n}}$ & 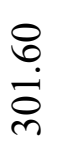 & $\begin{array}{l}\mathbb{J} \\
\ddot{0} \\
\tilde{\sigma}\end{array}$ & $\begin{array}{l}\infty \\
\infty \\
\text { ḋ } \\
\delta \\
0\end{array}$ & $\begin{array}{l}\text { Oे } \\
\text { ò } \\
\text { = }\end{array}$ & $\hat{\widehat{a}}$ & $\begin{array}{l}\hat{A} \\
\text { in } \\
\hat{\theta}\end{array}$ & $\begin{array}{l}\infty \\
\infty \\
\infty \\
\varpi \\
n\end{array}$ & $\begin{array}{l}\vec{\infty} \\
\dot{0}\end{array}$ & $\begin{array}{l}\infty \\
\infty \\
\infty\end{array}$ & $\begin{array}{l}\widetilde{0} \\
\stackrel{\text { I }}{ }\end{array}$ & $\begin{array}{l}\hat{n} \\
\text { ñ } \\
=\end{array}$ & 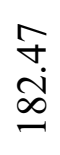 \\
\hline 3 & $\begin{array}{l}\underbrace{}_{1} \\
\end{array}$ & 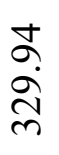 & $\begin{array}{l}\dddot{n} \\
\stackrel{2}{0} \\
0\end{array}$ & $\begin{array}{l}\hat{R} \\
\stackrel{0}{0}\end{array}$ & $\begin{array}{l}\hat{o} . \\
\stackrel{8}{O} \\
=\end{array}$ & $\frac{n}{\frac{n}{\infty}}$ & $\begin{array}{l}\hat{\beta} \\
\infty \\
0 \\
0\end{array}$ & $\overrightarrow{\hat{a}}$ & $\stackrel{n}{n}$ & ڤั & $\begin{array}{l}\stackrel{v}{n} \\
\stackrel{\sim}{\sim}\end{array}$ & $\begin{array}{l}\text { ָ̣ } \\
\stackrel{y}{ \pm}\end{array}$ & $\begin{array}{l}\hat{\sigma} \\
\text { ๙̇ } \\
\text { }\end{array}$ \\
\hline 4 & $\begin{array}{l}\overrightarrow{+} \\
\dot{t}\end{array}$ & $\begin{array}{l}\stackrel{n}{n} \\
\text { ñ } \\
\text { ñ }\end{array}$ & $\begin{array}{l}\vec{\nabla} \\
\infty \\
\sigma \\
0\end{array}$ & $\begin{array}{l}2 \\
\infty \\
\curvearrowright \\
\approx\end{array}$ & $\begin{array}{l}\hat{\Omega} \\
\Xi \\
\Xi\end{array}$ & $\begin{array}{l}\text { సે. } \\
ٌ \\
ٌ \\
\infty\end{array}$ & 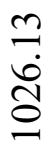 & $\begin{array}{l}\infty \\
\infty \\
i \\
i n\end{array}$ & $\frac{\Xi}{\sim}$ & $\underset{\infty}{\infty}$ & 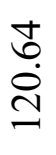 & $\begin{array}{l}\stackrel{\Xi}{\sigma} \\
\stackrel{\Im}{ \pm}\end{array}$ & $\begin{array}{l}\stackrel{\vartheta}{+} \\
\text { } \\
\stackrel{\sigma}{ }\end{array}$ \\
\hline 5 & $\stackrel{\stackrel{L}{1}}{\mathrm{~N}}$ & 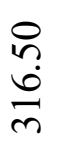 & $\begin{array}{l}0 \\
\infty \\
\infty \\
0\end{array}$ & $\begin{array}{l}\stackrel{0}{n} \\
\stackrel{0}{6} \\
0\end{array}$ & $\frac{8}{\stackrel{\circ}{\circ}}$ & $\frac{\circ}{\infty}$ & $\begin{array}{l}\stackrel{8}{n} \\
\frac{\hat{\sigma}}{0} \\
0\end{array}$ & $\begin{array}{l}\frac{n}{2} \\
\text { ลे } \\
\text { ñ }\end{array}$ & $\begin{array}{l}\infty \\
\infty \\
0 \\
0\end{array}$ & $\stackrel{\Omega}{\Omega}$ & 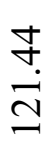 & 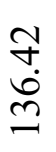 & $\begin{array}{l}\text { ป̄ } \\
\text { ஸ் } \\
\infty\end{array}$ \\
\hline 6 & $\frac{I}{\dot{y}}$ & 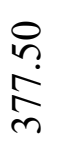 & $\begin{array}{l}\hat{\sigma} \\
\hat{\sigma} \\
\underline{0}\end{array}$ & $\begin{array}{l}\frac{0}{2} \\
\frac{2}{n} \\
\text { n }\end{array}$ & $\begin{array}{l}\hat{n} \\
\text { g̊ } \\
=\end{array}$ & $\begin{array}{l}n \\
n \\
\tilde{\infty} \\
\infty\end{array}$ & 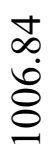 & $\begin{array}{l}\text { กุ' } \\
\text { oे }\end{array}$ & $\hat{\sigma}$ & $\stackrel{n}{n}$ & $\hat{a}$ & $\begin{array}{l}\stackrel{ }{\Xi} \\
\underset{J}{ \pm}\end{array}$ & $\begin{array}{l}\stackrel{+}{n} \\
\text { ๙̊}\end{array}$ \\
\hline 7 & $\sum_{\substack{1 \\
\sim}}^{0}$ & $\begin{array}{l}\overrightarrow{0} \\
\underset{\sim}{\Delta}\end{array}$ & \begin{tabular}{l}
0 \\
$n$ \\
\hdashline \\
0 \\
0
\end{tabular} & $\begin{array}{l}\hat{\sigma} \\
\dot{\delta}\end{array}$ & $\begin{array}{l}\vec{y} \\
\tilde{o} \\
ٍ \\
\exists\end{array}$ & $\begin{array}{l}\stackrel{\Omega}{\alpha} \\
\hat{b} \\
\infty\end{array}$ & $\begin{array}{l}\text { ¿े. } \\
\text { }\end{array}$ & $\begin{array}{l} \pm \\
\stackrel{\Xi}{n}\end{array}$ & $\stackrel{\infty}{\longrightarrow}$ & $\begin{array}{l}\infty \\
\infty \\
\infty\end{array}$ & $\begin{array}{l}\stackrel{n}{n} \\
\stackrel{n}{n}\end{array}$ & $\begin{array}{l}\text { ণิ } \\
\text { ஸे }\end{array}$ & $\begin{array}{l}\text { mె } \\
\infty \\
\infty\end{array}$ \\
\hline 8 & $\sum_{\substack{++}}^{0}$ & $\frac{\stackrel{q}{\dot{m}}}{m}$ & $\frac{\infty}{\stackrel{\infty}{\sigma}}$ & $\begin{array}{l}\text { ते } \\
\stackrel{0}{0} \\
\stackrel{n}{n}\end{array}$ & $\begin{array}{l}\stackrel{\infty}{\sigma} \underset{b}{\Xi} \\
=\end{array}$ & $\begin{array}{l}\stackrel{0}{\Omega} \\
\text { r) }\end{array}$ & 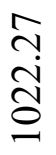 & $\frac{\mathscr{\infty}}{\dot{a}}$ & $\stackrel{1}{O}$ & $\begin{array}{l}\sigma \\
-\end{array}$ & $\begin{array}{l}\infty \\
\infty \\
\infty \\
=\end{array}$ & $\begin{array}{l}\text { ㄱ. } \\
\text { }\end{array}$ & $\begin{array}{c}\stackrel{N}{\sigma} \\
\stackrel{\sigma}{\sigma}\end{array}$ \\
\hline 9 & 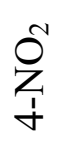 & $\begin{array}{l}\text { nै } \\
\frac{n}{n}\end{array}$ & مِ & $\begin{array}{l}n \\
\infty \\
\text { i } \\
\delta \\
0\end{array}$ & $\begin{array}{l}\text { مे } \\
\ddot{\infty} \\
\infty \\
0\end{array}$ & $\begin{array}{l}\overline{6} \\
\ddot{\circ} \\
\infty \\
\infty\end{array}$ & $\begin{array}{l}\stackrel{0}{n} \\
\stackrel{+}{0} \\
0\end{array}$ & $\begin{array}{l}\stackrel{n}{n} \\
\infty \\
\stackrel{N}{n} \\
n\end{array}$ & $\stackrel{m}{\longrightarrow}$ & $\vec{\infty}$ & $\begin{array}{l}\text { I } \\
\stackrel{\dot{J}}{\mathrm{~J}}\end{array}$ & $\begin{array}{l}\stackrel{\infty}{+} \\
\underset{+}{+}\end{array}$ & $\begin{array}{l}0 \\
+ \\
\dot{+} \\
\stackrel{-}{2}\end{array}$ \\
\hline
\end{tabular}


Table 3. Results of statistical analysis of infrared $v\left(\mathrm{~cm}^{-1}\right) \mathrm{CO}_{s-i s}, \mathrm{CO}_{s-\text { trans }}, \mathrm{CH}_{i p}, \mathrm{CH}_{o p}$,

$\mathrm{CH}=\mathrm{CH}_{o p}, \mathrm{C}=\mathrm{C}_{o p} \mathrm{NMR}$ chemical shifts $(\delta, \mathrm{ppm})$ of Protons and carbons of substituted 2-hydroxyphenyl-styrylketones with Hammett $\sigma, \sigma^{+}, \sigma_{\mathrm{I}} \sigma_{\mathrm{R}}$ constants and $\mathrm{F}$ and R parameters.

\begin{tabular}{|c|c|c|c|c|c|c|c|}
\hline Frequency & Constants & $\mathbf{r}$ & I & $\boldsymbol{\rho}$ & $\mathbf{S}$ & $\mathbf{n}$ & Correlated derivatives \\
\hline \multirow[t]{6}{*}{$\lambda_{\max }$} & $\sigma$ & 0.902 & 329.66 & -12.47 & 21.88 & 7 & $\begin{array}{c}\mathrm{H}, 3-\mathrm{Cl}, 4-\mathrm{Cl}, 2-\mathrm{F}, 2-\mathrm{OCH}_{3}, 4-\mathrm{OCH}_{3}, \\
\text { 4- } \mathrm{NO}_{2}\end{array}$ \\
\hline & $\sigma^{+}$ & 0.903 & 329.82 & -15.85 & 20.66 & 8 & $\begin{array}{c}\mathrm{H}, 2-\mathrm{Br}, 4-\mathrm{Cl}, 2-\mathrm{F}, 4-\mathrm{F}, 2-\mathrm{OCH}_{3} \\
\text { 4-OCH } \\
\end{array}$ \\
\hline & $\sigma_{\mathrm{I}}$ & 0.812 & 321.59 & 15.85 & 22.14 & 9 & $\begin{array}{c}\mathrm{H}, 2-\mathrm{Br}, 3-\mathrm{Cl}, 4-\mathrm{Cl}, 2-\mathrm{F}, 4-\mathrm{F}, 2-\mathrm{OCH}_{3}, \\
\text { 4- } \mathrm{OCH}_{3}, 4-\mathrm{NO}_{2}\end{array}$ \\
\hline & $\sigma_{\mathrm{R}}$ & 0.831 & 320.25 & -28.59 & 21.08 & 9 & $\begin{array}{c}\mathrm{H}, 2-\mathrm{Br}, 3-\mathrm{Cl}, 4-\mathrm{Cl}, 2-\mathrm{F}, 4-\mathrm{F}, 2-\mathrm{OCH}_{3}, \\
\text { 4- } \mathrm{OCH}_{3}, 4-\mathrm{NO}_{2}\end{array}$ \\
\hline & $\mathrm{F}$ & 0.824 & 317.13 & 24.38 & 21.47 & 9 & $\begin{array}{c}\mathrm{H}, 2-\mathrm{Br}, 3-\mathrm{Cl}, 4-\mathrm{Cl}, 2-\mathrm{F}, 4-\mathrm{F}, 2-\mathrm{OCH}_{3}, \\
\text { 4- } \mathrm{OCH}_{3}, 4-\mathrm{NO}_{2}\end{array}$ \\
\hline & $\mathrm{R}$ & 0.841 & 318.22 & -31.44 & 20.32 & 9 & $\begin{array}{c}\mathrm{H}, 2-\mathrm{Br}, 3-\mathrm{Cl}, 4-\mathrm{Cl}, 2-\mathrm{F}, 4-\mathrm{F}, 2-\mathrm{OCH}_{3}, \\
\text { 4- } \mathrm{OCH}_{3}, 4-\mathrm{NO}_{2}\end{array}$ \\
\hline \multirow[t]{6}{*}{$\mathrm{vCO}_{s-c i s}$} & $\sigma$ & 0.825 & 1651.43 & -3.74 & 23.49 & 9 & $\begin{array}{l}\mathrm{H}, 2-\mathrm{Br}, 3-\mathrm{Cl}, 4-\mathrm{Cl}, 2-\mathrm{F}, 4-\mathrm{F}, 2-\mathrm{OCH}_{3}, \\
\text { 4- } \mathrm{OCH}_{3}, 4-\mathrm{NO}_{2}\end{array}$ \\
\hline & $\sigma^{+}$ & 0.814 & 1650.46 & 3.84 & 23.44 & 9 & $\begin{array}{c}\mathrm{H}, 2-\mathrm{Br}, 3-\mathrm{Cl}, 4-\mathrm{Cl}, 2-\mathrm{F}, 4-\mathrm{F}, 2-\mathrm{OCH}_{3}, \\
\text { 4- } \mathrm{OCH}_{3}, 4-\mathrm{NO}_{2}\end{array}$ \\
\hline & $\sigma_{\mathrm{I}}$ & 0.792 & 1649.94 & 2.42 & 23.53 & 9 & $\begin{array}{c}\mathrm{H}, 2-\mathrm{Br}, 3-\mathrm{Cl}, 4-\mathrm{Cl}, 2-\mathrm{F}, 4-\mathrm{F}, 2-\mathrm{OCH}_{3}, \\
\text { 4- } \mathrm{OCH}_{3}, 4-\mathrm{NO}_{2}\end{array}$ \\
\hline & $\sigma_{\mathrm{R}}$ & 0.826 & 1644.57 & -23.52 & 22.71 & 9 & $\begin{array}{c}\mathrm{H}, 2-\mathrm{Br}, 3-\mathrm{Cl}, 4-\mathrm{Cl}, 2-\mathrm{F}, 4-\mathrm{F}, 2-\mathrm{OCH}_{3}, \\
\text { 4- } \mathrm{OCH}_{3}, 4-\mathrm{NO}_{2}\end{array}$ \\
\hline & $\mathrm{F}$ & 0.751 & 1650.89 & 0.07 & 23.53 & 9 & $\begin{array}{c}\mathrm{H}, 2-\mathrm{Br}, 3-\mathrm{Cl}, 4-\mathrm{Cl}, 2-\mathrm{F}, 4-\mathrm{F}, 2-\mathrm{OCH}_{3}, \\
\text { 4- } \mathrm{OCH}_{3}, 4-\mathrm{NO}_{2}\end{array}$ \\
\hline & $\mathrm{R}$ & 0.861 & 1646.37 & -14.66 & 23.12 & 9 & $\begin{array}{c}\mathrm{H}, 2-\mathrm{Br}, 3-\mathrm{Cl}, 4-\mathrm{Cl}, 2-\mathrm{F}, 4-\mathrm{F}, 2-\mathrm{OCH}_{3}, \\
\text { 4- } \mathrm{OCH}_{3}, 4-\mathrm{NO}_{2}\end{array}$ \\
\hline \multirow[t]{6}{*}{$\mathrm{vCO}_{\text {s-trans }}$} & $\sigma$ & 0.904 & 1595.01 & 16.87 & 14.17 & 6 & 2-Br, 3-Cl, 4-Cl, 2-F, 4-F, 4-OCH 3 \\
\hline & $\sigma^{+}$ & 0.906 & 1595.07 & 18.85 & 11.58 & 6 & 2-Br, 3-Cl, 4-Cl, 2-F, 4-F, 4-OCH \\
\hline & $\sigma_{\mathrm{I}}$ & 0.805 & 1595.46 & 4.58 & 15.49 & 9 & $\begin{array}{c}\mathrm{H}, 2-\mathrm{Br}, 3-\mathrm{Cl}, 4-\mathrm{Cl}, 2-\mathrm{F}, 4-\mathrm{F}, 2-\mathrm{OCH}_{3}, \\
\text { 4- } \mathrm{OCH}_{3}, 4-\mathrm{NO}_{2}\end{array}$ \\
\hline & $\sigma_{\mathrm{R}}$ & 0.829 & 1602.03 & 17.52 & 14.82 & 9 & $\begin{array}{c}\mathrm{H}, 2-\mathrm{Br}, 3-\mathrm{Cl}, 4-\mathrm{Cl}, 2-\mathrm{F}, 4-\mathrm{F}, 2-\mathrm{OCH}_{3}, \\
\text { 4- } \mathrm{OCH}_{3}, 4-\mathrm{NO}_{2}\end{array}$ \\
\hline & $\mathrm{F}$ & 0.804 & 1597.32 & -0.04 & 14.1 & 9 & $\begin{array}{c}\mathrm{H}, 2-\mathrm{Br}, 3-\mathrm{Cl}, 4-\mathrm{Cl}, 2-\mathrm{F}, 4-\mathrm{F}, 2-\mathrm{OCH}_{3}, \\
\text { 4- } \mathrm{OCH}_{3}, 4-\mathrm{NO}_{2}\end{array}$ \\
\hline & $\mathrm{R}$ & 0.907 & 1604.03 & 21.71 & 14.1 & 7 & $\begin{array}{c}\mathrm{H}, 2-\mathrm{Br}, 3-\mathrm{Cl}, 4-\mathrm{Cl}, 2-\mathrm{F}, 2-\mathrm{OCH}_{3} \\
\text { 4- } \mathrm{NO}_{2}\end{array}$ \\
\hline \multirow[t]{6}{*}{${ } \mathrm{CH}_{i p}$} & $\sigma$ & 0.904 & 1113.66 & -20.91 & 16.63 & 7 & $\begin{array}{c}\mathrm{H}, 2-\mathrm{Br}, 3-\mathrm{Cl}, 4-\mathrm{Cl}, 2-\mathrm{F}, 4-\mathrm{OCH}_{3}, \\
\text { 4-NO } \\
\end{array}$ \\
\hline & $\sigma^{+}$ & 0.846 & 1112.67 & -15.60 & 16.27 & 9 & $\begin{array}{c}\mathrm{H}, 2-\mathrm{Br}, 3-\mathrm{Cl}, 4-\mathrm{Cl}, 2-\mathrm{F}, 4-\mathrm{F}, 2-\mathrm{OCH}_{3}, \\
\text { 4- } \mathrm{OCH}_{3}, 4-\mathrm{NO}_{2}\end{array}$ \\
\hline & $\sigma_{\mathrm{I}}$ & 0.809 & 1114.21 & -8.43 & 18.3 & 9 & $\begin{array}{c}\mathrm{H}, 2-\mathrm{Br}, 3-\mathrm{Cl}, 4-\mathrm{Cl}, 2-\mathrm{F}, 4-\mathrm{F}, 2-\mathrm{OCH}_{3}, \\
\text { 4- } \mathrm{OCH}_{3}, 4-\mathrm{NO}_{2}\end{array}$ \\
\hline & $\sigma_{\mathrm{R}}$ & 0.804 & 1102.40 & -31.18 & 16.47 & 9 & $\begin{array}{c}\mathrm{H}, 2-\mathrm{Br}, 3-\mathrm{Cl}, 4-\mathrm{Cl}, 2-\mathrm{F}, 4-\mathrm{F}, 2-\mathrm{OCH}_{3}, \\
\text { 4- } \mathrm{OCH}_{3}, 4-\mathrm{NO}_{2}\end{array}$ \\
\hline & $\mathrm{F}$ & 0.807 & 1108.56 & 5.01 & 18.33 & 9 & $\begin{array}{c}\mathrm{H}, 2-\mathrm{Br}, 3-\mathrm{Cl}, 4-\mathrm{Cl}, 2-\mathrm{F}, 4-\mathrm{F}, 2-\mathrm{OCH}_{3}, \\
\text { 4- } \mathrm{OCH}_{3}, 4-\mathrm{NO}_{2}\end{array}$ \\
\hline & $\mathrm{R}$ & 0.748 & 1101.52 & -30.00 & 16.07 & 9 & $\begin{array}{c}\mathrm{H}, 2-\mathrm{Br}, 3-\mathrm{Cl}, 4-\mathrm{Cl}, 2-\mathrm{F}, 4-\mathrm{F}, 2-\mathrm{OCH}_{3}, \\
\text { 4- } \mathrm{OCH}_{3}, 4-\mathrm{NO}_{2}\end{array}$ \\
\hline \multirow[t]{6}{*}{$v \mathrm{CH}_{o p}$} & $\sigma$ & 0.902 & 849.97 & 12.76 & 21.21 & 7 & $\mathrm{H}, 2-\mathrm{Br}, 3-\mathrm{Cl}, 4-\mathrm{Cl}, 2-\mathrm{F}, 4-\mathrm{F}, 4-\mathrm{OCH}_{3}$ \\
\hline & $\sigma^{+}$ & 0.904 & 849.58 & 17.84 & 19.42 & 7 & $\mathrm{H}, 2-\mathrm{Br}, 3-\mathrm{Cl}, 4-\mathrm{Cl}, 2-\mathrm{F}, 4-\mathrm{F}, 4-\mathrm{OCH}_{3}$ \\
\hline & $\sigma_{\mathrm{I}}$ & 0.801 & 855.18 & 8.65 & 21.67 & 9 & $\begin{array}{c}\mathrm{H}, 2-\mathrm{Br}, 3-\mathrm{Cl}, 4-\mathrm{Cl}, 2-\mathrm{F}, 4-\mathrm{F}, 2-\mathrm{OCH}_{3}, \\
\text { 4- } \mathrm{OCH}_{3}, 4-\mathrm{NO}_{2}\end{array}$ \\
\hline & $\sigma_{\mathrm{R}}$ & 0.875 & 857.85 & 22.78 & 20.91 & 9 & $\begin{array}{c}\mathrm{H}, 2-\mathrm{Br}, 3-\mathrm{Cl}, 4-\mathrm{Cl}, 2-\mathrm{F}, 4-\mathrm{F}, 2-\mathrm{OCH}_{3}, \\
\text { 4- } \mathrm{OCH}_{3}, 4-\mathrm{NO}_{2}\end{array}$ \\
\hline & $\mathrm{F}$ & 0.785 & 861.96 & -23.10 & 20.91 & 9 & $\begin{array}{c}\mathrm{H}, 2-\mathrm{Br}, 3-\mathrm{Cl}, 4-\mathrm{Cl}, 2-\mathrm{F}, 4-\mathrm{F}, 2-\mathrm{OCH}_{3}, \\
\text { 4- } \mathrm{OCH}_{3}, 4-\mathrm{NO}_{2}\end{array}$ \\
\hline & $\mathrm{R}$ & 0.847 & 862.05 & 33.39 & 19.34 & 9 & $\begin{array}{c}\mathrm{H}, 2-\mathrm{Br}, 3-\mathrm{Cl}, 4-\mathrm{Cl}, 2-\mathrm{F}, 4-\mathrm{F}, 2-\mathrm{OCH}_{3}, \\
\text { 4- } \mathrm{OCH}_{3}, 4-\mathrm{NO}_{2}\end{array}$ \\
\hline$v \mathrm{C}=\mathrm{CH}_{o p}$ & $\sigma$ & 0.816 & 1024.54 & 13.30 & 30.39 & 9 & $\begin{array}{c}\mathrm{H}, 2-\mathrm{Br}, 3-\mathrm{Cl}, 4-\mathrm{Cl}, 2-\mathrm{F}, 4-\mathrm{F}, 2-\mathrm{OCH}_{3}, \\
\text { 4- } \mathrm{OCH}_{3}, 4-\mathrm{NO}_{2}\end{array}$ \\
\hline
\end{tabular}




\begin{tabular}{|c|c|c|c|c|c|c|c|}
\hline & $\sigma^{+}$ & 0.831 & 1023.87 & 20.82 & 28.60 & 9 & $\begin{array}{c}\mathrm{H}, 2-\mathrm{Br}, 3-\mathrm{Cl}, 4-\mathrm{Cl}, 2-\mathrm{F}, 4-\mathrm{F}, 2-\mathrm{OCH}_{3}, \\
\text { 4- } \mathrm{OCH}_{3}, 4-\mathrm{NO}_{2}\end{array}$ \\
\hline & $\sigma_{\mathrm{I}}$ & 0.84 & 1019.82 & 16.21 & 30.62 & 9 & $\begin{array}{c}\mathrm{H}, 2-\mathrm{Br}, 3-\mathrm{Cl}, 4-\mathrm{Cl}, 2-\mathrm{F}, 4-\mathrm{F}, 2-\mathrm{OCH}_{3}, \\
\text { 4- } \mathrm{OCH}_{3}, 4-\mathrm{NO}_{2}\end{array}$ \\
\hline & $\sigma_{\mathrm{R}}$ & 0.816 & 1021.22 & -18.97 & 30.39 & 9 & $\begin{array}{c}\mathrm{H}, 2-\mathrm{Br}, 3-\mathrm{Cl}, 4-\mathrm{Cl}, 2-\mathrm{F}, 4-\mathrm{F}, 2-\mathrm{OCH}_{3}, \\
\text { 4- } \mathrm{OCH}_{3}, 4-\mathrm{NO}_{2}\end{array}$ \\
\hline & $\mathrm{F}$ & 0.733 & 1008.29 & 40.61 & 28.97 & 9 & $\begin{array}{c}\mathrm{H}, 2-\mathrm{Br}, 3-\mathrm{Cl}, 4-\mathrm{Cl}, 2-\mathrm{F}, 4-\mathrm{F}, 2-\mathrm{OCH}_{3}, \\
\text { 4- } \mathrm{OCH}_{3}, 4-\mathrm{NO}_{2}\end{array}$ \\
\hline & $\mathrm{R}$ & 0.823 & 1018.95 & -23.84 & 29.97 & 9 & $\begin{array}{c}\mathrm{H}, 2-\mathrm{Br}, 3-\mathrm{Cl}, 4-\mathrm{Cl}, 2-\mathrm{F}, 4-\mathrm{F}, 2-\mathrm{OCH}_{3}, \\
\text { 4- } \mathrm{OCH}_{3}, 4-\mathrm{NO}_{2}\end{array}$ \\
\hline \multirow[t]{6}{*}{$v \mathrm{C}=\mathrm{C}_{o p}$} & $\sigma$ & 0.844 & 527.79 & 41.56 & 30.87 & 9 & $\begin{array}{c}\mathrm{H}, 2-\mathrm{Br}, 3-\mathrm{Cl}, 4-\mathrm{Cl}, 2-\mathrm{F}, 4-\mathrm{F}, 2-\mathrm{OCH}_{3}, \\
\text { 4- } \mathrm{OCH}_{3}, 4-\mathrm{NO}_{2}\end{array}$ \\
\hline & $\sigma^{+}$ & 0.905 & 529.18 & 35.84 & 28.44 & 7 & $\begin{array}{c}\mathrm{H}, 2-\mathrm{Br}, 3-\mathrm{Cl}, 4-\mathrm{F}, 2-\mathrm{OCH}_{3}, 4-\mathrm{OCH}_{3}, \\
\text { 4- } \mathrm{NO}_{2}\end{array}$ \\
\hline & $\sigma_{\mathrm{I}}$ & 0.812 & 513.31 & 50.00 & 33.04 & 9 & $\begin{array}{c}\mathrm{H}, 2-\mathrm{Br}, 3-\mathrm{Cl}, 4-\mathrm{Cl}, 2-\mathrm{F}, 4-\mathrm{F}, 2-\mathrm{OCH}_{3}, \\
\text { 4- } \mathrm{OCH}_{3}, 4-\mathrm{NO}_{2}\end{array}$ \\
\hline & $\sigma_{\mathrm{R}}$ & 0.81 & 537.33 & 14.46 & 34.35 & 9 & $\begin{array}{c}\mathrm{H}, 2-\mathrm{Br}, 3-\mathrm{Cl}, 4-\mathrm{Cl}, 2-\mathrm{F}, 4-\mathrm{F}, 2-\mathrm{OCH}_{3}, \\
\text { 4- } \mathrm{OCH}_{3}, 4-\mathrm{NO}_{2}\end{array}$ \\
\hline & $\mathrm{F}$ & 0.833 & 513.61 & 44.57 & 32.6 & 9 & $\begin{array}{c}\mathrm{H}, 2-\mathrm{Br}, 3-\mathrm{Cl}, 4-\mathrm{Cl}, 2-\mathrm{F}, 4-\mathrm{F}, 2-\mathrm{OCH}_{3}, \\
\text { 4- } \mathrm{OCH}_{3}, 4-\mathrm{NO}_{2}\end{array}$ \\
\hline & $\mathrm{R}$ & 0.809 & 536.94 & 11.36 & 34.4 & 9 & $\begin{array}{c}\mathrm{H}, 2-\mathrm{Br}, 3-\mathrm{Cl}, 4-\mathrm{Cl}, 2-\mathrm{F}, 4-\mathrm{F}, 2-\mathrm{OCH}_{3}, \\
\text { 4- } \mathrm{OCH}_{3}, 4-\mathrm{NO}_{2}\end{array}$ \\
\hline \multirow[t]{6}{*}{$\delta_{\mathrm{H} \alpha}$} & $\sigma$ & 0.703 & 7.11 & -0.02 & 0.27 & 9 & $\begin{array}{c}\mathrm{H}, 2-\mathrm{Br}, 3-\mathrm{Cl}, 4-\mathrm{Cl}, 2-\mathrm{F}, 4-\mathrm{F}, 2-\mathrm{OCH}_{3}, \\
\text { 4- } \mathrm{OCH}_{3}, 4-\mathrm{NO}_{2}\end{array}$ \\
\hline & $\sigma^{+}$ & 0.685 & 7.12 & -0.06 & 0.26 & 9 & $\begin{array}{c}\mathrm{H}, 2-\mathrm{Br}, 3-\mathrm{Cl}, 4-\mathrm{Cl}, 2-\mathrm{F}, 4-\mathrm{F}, 2-\mathrm{OCH}_{3}, \\
\text { 4- } \mathrm{OCH}_{3}, 4-\mathrm{NO}_{2}\end{array}$ \\
\hline & $\sigma_{\mathrm{I}}$ & 0.814 & 7.34 & -0.57 & 0.24 & 9 & $\begin{array}{c}\mathrm{H}, 2-\mathrm{Br}, 3-\mathrm{Cl}, 4-\mathrm{Cl}, 2-\mathrm{F}, 4-\mathrm{F}, 2-\mathrm{OCH}_{3}, \\
\text { 4- } \mathrm{OCH}_{3}, 4-\mathrm{NO}_{2}\end{array}$ \\
\hline & $\sigma_{\mathrm{R}}$ & 0.831 & 7.20 & 0.33 & 0.25 & 9 & $\begin{array}{c}\mathrm{H}, 2-\mathrm{Br}, 3-\mathrm{Cl}, 4-\mathrm{Cl}, 2-\mathrm{F}, 4-\mathrm{F}, 2-\mathrm{OCH}_{3}, \\
\text { 4- } \mathrm{OCH}_{3}, 4-\mathrm{NO}_{2}\end{array}$ \\
\hline & $\mathrm{F}$ & 0.905 & 7.38 & -0.60 & 0.22 & 8 & $\begin{array}{c}\mathrm{H}, 2-\mathrm{Br}, 4-\mathrm{Cl}, 2-\mathrm{F}, 4-\mathrm{F}, 2-\mathrm{OCH}_{3} \\
\text { 4-OCH } \\
3,4-\mathrm{NO}_{2}\end{array}$ \\
\hline & $\mathrm{R}$ & 0.834 & 7.21 & 0.33 & 0.25 & 9 & $\begin{array}{c}\mathrm{H}, 2-\mathrm{Br}, 3-\mathrm{Cl}, 4-\mathrm{Cl}, 2-\mathrm{F}, 4-\mathrm{F}, 2-\mathrm{OCH}_{3}, \\
\text { 4-OCH }, 4-\mathrm{NO}_{2}\end{array}$ \\
\hline \multirow[t]{6}{*}{$\delta_{\mathrm{H} \beta}$} & $\sigma$ & 0.905 & 7.89 & 0.38 & 0.2 & 7 & $\begin{array}{c}\mathrm{H}, 3-\mathrm{Cl}, 4-\mathrm{Cl}, 2-\mathrm{F}, 2-\mathrm{OCH}_{3}, 4-\mathrm{OCH}_{3}, \\
\text { 4- } \mathrm{NO}_{2}\end{array}$ \\
\hline & $\sigma^{+}$ & 0.906 & 7.91 & 0.29 & 0.18 & 7 & $\begin{array}{c}\mathrm{H}, 3-\mathrm{Cl}, 4-\mathrm{Cl}, 2-\mathrm{F}, 2-\mathrm{OCH}_{3}, 4-\mathrm{OCH}_{3}, \\
\text { 4- } \mathrm{NO}_{2}\end{array}$ \\
\hline & $\sigma_{\mathrm{I}}$ & 0.816 & 7.84 & 0.24 & 0.24 & 9 & $\begin{array}{c}\mathrm{H}, 2-\mathrm{Br}, 3-\mathrm{Cl}, 4-\mathrm{Cl}, 2-\mathrm{F}, 4-\mathrm{F}, 2-\mathrm{OCH}_{3}, \\
\text { 4- } \mathrm{OCH}_{3}, 4-\mathrm{NO}_{2}\end{array}$ \\
\hline & $\sigma_{R}$ & 0.905 & 8.09 & 0.56 & 0.19 & 7 & $\begin{array}{c}\mathrm{H}, 2-\mathrm{Br}, 3-\mathrm{Cl}, 4-\mathrm{Cl}, 4-\mathrm{F}, 4-\mathrm{OCH}_{3}, \\
\text { 4- } \mathrm{NO}_{2}\end{array}$ \\
\hline & $\mathrm{F}$ & 0.725 & 7.95 & -0.25 & 0.24 & 9 & $\begin{array}{c}\mathrm{H}, 2-\mathrm{Br}, 3-\mathrm{Cl}, 4-\mathrm{Cl}, 2-\mathrm{F}, 4-\mathrm{F}, \\
2-\mathrm{OCH}_{3}, 4-\mathrm{OCH}_{3}, 4-\mathrm{NO}_{2}\end{array}$ \\
\hline & $\mathrm{R}$ & 0.906 & 8.12 & 0.57 & 0.18 & 7 & $\begin{array}{c}\mathrm{H}, 2-\mathrm{Br}, 3-\mathrm{Cl}, 4-\mathrm{Cl}, 4-\mathrm{F}, 4-\mathrm{OCH}_{3}, \\
4-\mathrm{NO}_{2}\end{array}$ \\
\hline \multirow[t]{6}{*}{$\delta \mathrm{CO}$} & $\sigma$ & 0.825 & 189.21 & 3.92 & 5.57 & 9 & $\begin{array}{c}\mathrm{H}, 2-\mathrm{Br}, 3-\mathrm{Cl}, 4-\mathrm{Cl}, 2-\mathrm{F}, 4-\mathrm{F}, 2-\mathrm{OCH}_{3}, \\
\text { 4- } \mathrm{OCH}_{3}, 4-\mathrm{NO}_{2}\end{array}$ \\
\hline & $\sigma^{+}$ & 0.815 & 189.92 & -1.54 & 5.7 & 9 & $\begin{array}{c}\mathrm{H}, 2-\mathrm{Br}, 3-\mathrm{Cl}, 4-\mathrm{Cl}, 2-\mathrm{F}, 4-\mathrm{F}, 2-\mathrm{OCH}_{3}, \\
\text { 4-OCH }{ }_{3}, 4-\mathrm{NO}_{2}\end{array}$ \\
\hline & $\sigma_{\mathrm{I}}$ & 0.803 & 189.35 & 0.97 & 5.76 & 9 & $\begin{array}{c}\mathrm{H}, 2-\mathrm{Br}, 3-\mathrm{Cl}, 4-\mathrm{Cl}, 2-\mathrm{F}, 4-\mathrm{F}, 2-\mathrm{OCH}_{3}, \\
\text { 4- } \mathrm{OCH}_{3}, 4-\mathrm{NO}_{2}\end{array}$ \\
\hline & $\sigma_{\mathrm{R}}$ & 0.508 & 192.72 & 11.06 & 4.98 & 9 & $\begin{array}{c}\mathrm{H}, 2-\mathrm{Br}, 3-\mathrm{Cl}, 4-\mathrm{Cl}, 2-\mathrm{F}, 4-\mathrm{F}, 2-\mathrm{OCH}_{3}, \\
\text { 4- } \mathrm{OCH}_{3}, 4-\mathrm{NO}_{2}\end{array}$ \\
\hline & $\mathrm{F}$ & 0.809 & 190.71 & -2.18 & 5.74 & 9 & $\begin{array}{c}\mathrm{H}, 2-\mathrm{Br}, 3-\mathrm{Cl}, 4-\mathrm{Cl}, 2-\mathrm{F}, 4-\mathrm{F}, 2-\mathrm{OCH}_{3}, \\
\text { 4- } \mathrm{OCH}_{3}, 4-\mathrm{NO}_{2}\end{array}$ \\
\hline & $\mathrm{R}$ & 0.838 & 192.06 & 7.49 & 5.31 & 9 & $\begin{array}{c}\mathrm{H}, 2-\mathrm{Br}, 3-\mathrm{Cl}, 4-\mathrm{Cl}, 2-\mathrm{F}, 4-\mathrm{F}, 2-\mathrm{OCH}_{3}, \\
\text { 4- } \mathrm{OCH}_{3}, 4-\mathrm{NO}_{2}\end{array}$ \\
\hline \multirow[t]{5}{*}{$\delta \mathrm{C} \alpha$} & $\sigma$ & 0.904 & 121.41 & 2.72 & 1.81 & 8 & $\begin{array}{c}\mathrm{H}, 2-\mathrm{Br}, 3-\mathrm{Cl}, 4-\mathrm{Cl}, 2-\mathrm{F}, 4-\mathrm{F}, 4-\mathrm{OCH}_{3}, \\
\text { 4- } \mathrm{NO}_{2}\end{array}$ \\
\hline & $\sigma^{+}$ & 0.905 & 121.53 & 2.11 & 1.73 & 7 & $\begin{array}{c}\mathrm{H}, 2-\mathrm{Br}, 4-\mathrm{Cl}, 2-\mathrm{F}, 2-\mathrm{OCH}_{3}, 4-\mathrm{OCH}_{3}, \\
\text { 4- } \mathrm{NO}_{2}\end{array}$ \\
\hline & $\sigma_{\mathrm{I}}$ & 0.833 & 120.45 & 3.30 & 1.97 & 9 & $\begin{array}{c}\mathrm{H}, 2-\mathrm{Br}, 3-\mathrm{Cl}, 4-\mathrm{Cl}, 2-\mathrm{F}, 4-\mathrm{F} \\
2-\mathrm{OCH}_{3}, 4-\mathrm{OCH}_{3}, 4-\mathrm{NO}_{2}\end{array}$ \\
\hline & $\sigma_{R}$ & 0.835 & 122.50 & 2.68 & 1.96 & 9 & $\begin{array}{l}\mathrm{H}, 2-\mathrm{Br}, 3-\mathrm{Cl}, 4-\mathrm{Cl}, 2-\mathrm{F}, 4-\mathrm{F}, \\
2-\mathrm{OCH}_{3}, 4-\mathrm{OCH}_{3}, 4-\mathrm{NO}_{2}\end{array}$ \\
\hline & $\mathrm{F}$ & 0.809 & 121.43 & 0.79 & 2.07 & 9 & $\begin{array}{l}\mathrm{H}, 2-\mathrm{Br}, 3-\mathrm{Cl}, 4-\mathrm{Cl}, 2-\mathrm{F}, 4-\mathrm{F}, \\
2-\mathrm{OCH}_{3}, 4-\mathrm{OCH}_{3}, 4-\mathrm{NO}_{2}\end{array}$ \\
\hline
\end{tabular}




\begin{tabular}{|c|c|c|c|c|c|c|c|}
\hline & $\mathrm{R}$ & 0.844 & 122.76 & 3.15 & 1.86 & 9 & $\begin{array}{c}\mathrm{H}, 2-\mathrm{Br}, 3-\mathrm{Cl}, 4-\mathrm{Cl}, 2-\mathrm{F}, 4-\mathrm{F}, 2-\mathrm{OCH}_{3}, \\
\text { 4- }-\mathrm{OCH}_{3}, 4-\mathrm{NO}_{2}\end{array}$ \\
\hline \multirow[t]{6}{*}{$\delta C_{\beta}$} & $\sigma$ & 0.907 & 138.39 & 11.06 & 3.35 & 9 & $\begin{array}{c}\mathrm{H}, 2-\mathrm{Br}, 3-\mathrm{Cl}, 4-\mathrm{Cl}, 2-\mathrm{F}, 4-\mathrm{F}, 2-\mathrm{OCH}_{3}, \\
4-\mathrm{OCH}_{3}, 4-\mathrm{NO}_{2}\end{array}$ \\
\hline & $\sigma^{+}$ & 0.905 & 139.32 & 4.88 & 4.6 & 9 & $\begin{array}{c}\mathrm{H}, 2-\mathrm{Br}, 3-\mathrm{Cl}, 4-\mathrm{Cl}, 2-\mathrm{F}, 4-\mathrm{F}, 2-\mathrm{OCH}_{3}, \\
\text { 4- } \mathrm{OCH}_{3}, 4-\mathrm{NO}_{2}\end{array}$ \\
\hline & $\sigma_{\mathrm{I}}$ & 0.905 & 134.11 & 14.37 & 4.46 & 8 & $\begin{array}{l}\text { 2-Br, 3-Cl, 4-Cl, 2-F, 4-F, } \\
2-\mathrm{OCH}_{3}, 4-\mathrm{OCH}_{3}, 4-\mathrm{NO}_{2}\end{array}$ \\
\hline & $\sigma_{\mathrm{R}}$ & 0.903 & 143.45 & 13.18 & 4.06 & 9 & $\begin{array}{c}\mathrm{H}, 2-\mathrm{Br}, 3-\mathrm{Cl}, 4-\mathrm{Cl}, 2-\mathrm{F}, 4-\mathrm{F}, \\
2-\mathrm{OCH}_{3}, 4-\mathrm{OCH}_{3}, 4-\mathrm{NO}_{2}\end{array}$ \\
\hline & $\mathrm{F}$ & 0.836 & 136.23 & 8.25 & 4.88 & 9 & $\begin{array}{c}\mathrm{H}, 2-\mathrm{Br}, 3-\mathrm{Cl}, 4-\mathrm{Cl}, 2-\mathrm{F}, 4-\mathrm{F}, \\
2-\mathrm{OCH}_{3}, 4-\mathrm{OCH}_{3}, 4-\mathrm{NO}_{2}\end{array}$ \\
\hline & $\mathrm{R}$ & 0.905 & 142.99 & 9.98 & 4.42 & 8 & $\begin{array}{c}\mathrm{H}, 2-\mathrm{Br}, 3-\mathrm{Cl}, 4-\mathrm{Cl}, 2-\mathrm{F}, 2-\mathrm{OCH}_{3}, \\
\text { 4-OCH } 3 \text {, 4-NO } \mathrm{NO}_{2}\end{array}$ \\
\hline
\end{tabular}

$\mathrm{r}=$ correlation coefficient; $\rho=$ slope $; \mathrm{I}=$ intercept $; \mathrm{s}=$ standard deviation; $\mathrm{n}=$ number of substituents<smiles>[X]c1cccc(/C=C/C(=O)c2ccccc2O)c1</smiles>

$s$-cis

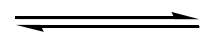

s-trans<smiles>[X]c1cccc(/C=C(\C=C\C(=O)c2ccccc2O)c2ccccc2)c1</smiles>

Fig. 2. The $s$-cis and s-trans conformers of substituted 2-hydroxyphenyl-styrylketones.

The results of single parameter statistical analysis of carbonyl frequencies of $s$-cis conformers with all Hammett substituent constants, F and R parameters were poor correlated The failure in correlation is due the conjugation between the substituent and the carbonyl group in chalcones. The $s$-trans conformer gave satisfactory correlation for $\sigma(\mathrm{r}=0.904), \sigma^{+}(\mathrm{r}=0.906)$ and $\mathrm{R}$ parameter $(\mathrm{r}=$ 0.907). Hammett constants $\sigma_{I}, \sigma_{R}$ and $\mathrm{F}$ parameter gave the poor correlation. The correlation of $\mathrm{CH}$ in-plane with Hammett substituent constants, $\mathrm{F}$ and $\mathrm{R}$ parameters were poor correlated and out of plane modes with Hammett $\sigma(\mathrm{r}=0.902), \sigma^{+}(\mathrm{r}=0.904)$ shown the satisfactory correltion. The $\sigma_{\mathrm{I}}, \sigma_{\mathrm{R}}$ constants and $\mathrm{F}$ and $\mathrm{R}$ parameters has poorly correlated. The $\mathrm{CH}=\mathrm{CH}$ out of plane modes with all Hammett substituent constants, $\mathrm{F}$ and $\mathrm{R}$ parameters were fail in correlation and the $\mathrm{C}=\mathrm{C}$ out of plane Hammett constant gave satisfactory correlation for $\sigma^{+}(\mathrm{r}=0.905)$. The results of poor correlation is due to the conjugation between the substituent and the vinyl group in chalcones as shown in Figure 1. 
In view of the inability of some of the $\sigma$ constants to produce individually satisfactory correlations, it was thought that worthwhile to seek multiple correlations involving either $\sigma_{I}$ and $\sigma_{R}$ constants or Swain-Lupton's [44] F and R parameters. The correlation equations for s-cis, s-trans and deformation modes are given in equations 5-16.

$$
\begin{aligned}
& v \mathrm{CO}_{s-c i s}\left(\mathrm{~cm}^{-1}\right)=1643.261( \pm 22.498)+3.218( \pm 1.740) \sigma_{\mathrm{I}}-23.587( \pm 7.452) \sigma_{\mathrm{R}} \\
& (R=0.926, \mathrm{n}=9, \mathrm{P}>90 \%) \\
& v \mathrm{CO}_{s-c i s}\left(\mathrm{~cm}^{-1}\right)=1648.215( \pm 19.117)-4.951( \pm 1.105) \mathrm{F}-15.835( \pm 5.949) \mathrm{R} \\
& (R=0.919, \mathrm{n}=9, \mathrm{P}>90 \%) \\
& v \mathrm{CO}_{\text {s-trans }}\left(\mathrm{cm}^{-1}\right)=1600.406( \pm 14.666)+3.990( \pm 1.817) \sigma_{\mathrm{I}}+17.440( \pm 5.111) \sigma_{\mathrm{R}} \\
& (R=0.929, \quad \mathrm{n}=9, \mathrm{P}>90 \%) \\
& v \mathrm{CO}_{s-\text { trans }}\left(\mathrm{cm}^{-1}\right)=1601.288( \pm 11.580)+7.397( \pm 2.081) \mathrm{F}+23.462( \pm 19.958) \mathrm{R} \\
& (R=0.943, \mathrm{n}=9, \mathrm{P}>90 \%) \\
& v \mathrm{CH}_{i p}\left(\mathrm{~cm}^{-1}\right)=1105.420( \pm 16.257)-7.386( \pm 2.051) \sigma_{\mathrm{I}}-31.030( \pm 25.617) \sigma_{\mathrm{R}} \\
& (R=0.945, \mathrm{n}=9, \mathrm{P}>90 \%) \\
& v \mathrm{CH}_{i p}\left(\mathrm{~cm}^{-1}\right)=1103.297( \pm 13.268)-4.768( \pm 1.446) \mathrm{F}+31.128( \pm 10.868) \mathrm{R} \\
& (R=0.948, \mathrm{n}=9, \mathrm{P}>90 \%) \\
& v \mathrm{CH}_{o p}\left(\mathrm{~cm}^{-1}\right)=861.694( \pm 20.633)-9.424( \pm 3.948) \sigma_{\mathrm{I}}+22.969( \pm 7.513) \sigma_{\mathrm{R}} \\
& (R=0.928, \quad \mathrm{n}=9, \mathrm{P}>90 \%) \\
& \nu \mathrm{CH}_{o p}\left(\mathrm{~cm}^{-1}\right)=867.073( \pm 15.769)-13.534( \pm 4.432) \mathrm{F}+30.179( \pm 27.179) \mathrm{R} \\
& (R=0.948, \quad \mathrm{n}=9, \quad \mathrm{P}>90 \%) \\
& v \mathrm{CH}=\mathrm{CH}_{o p}\left(\mathrm{~cm}^{-1}\right)=1014.349( \pm 29.925)+16.861( \pm 4.841) \sigma_{\mathrm{I}}-19.309( \pm 6.156) \sigma_{\mathrm{R}} \\
& (R=0.919, \quad \mathrm{n}=9, \mathrm{P}>90 \%) \\
& (R=0.936, \quad \mathrm{n}=9, \mathrm{P}>90 \%) \\
& v \mathrm{C}=\mathrm{C}_{o p}\left(\mathrm{~cm}^{-1}\right)=517.132( \pm 32.554)+49.542( \pm 13.187) \sigma_{\mathrm{I}}+13.452( \pm 4.210) \sigma_{\mathrm{R}} \\
& (R=0.931, \mathrm{n}=9, \mathrm{P}>90 \%) \\
& \nu \mathrm{C}=\mathrm{C}_{o p}\left(\mathrm{~cm}^{-1}\right)=517.132( \pm 26.401)+52.081( \pm 52.624) \mathrm{F}+23.702( \pm 7.504) \mathrm{R} \\
& (\mathrm{R}=0.938, \mathrm{n}=9, \mathrm{P}>90 \%)
\end{aligned}
$$

\section{1. 3. ${ }^{1} \mathrm{H}$ NMR spectral study}

Deuteriochloroform was used for recording the ${ }^{1} \mathrm{H}$ NMR spectra of synthesized chalcones employing tetramethylsilane (TMS) as internal standard. The ethylenic protons signals of the chalcones were assigned from their spectra. They were calculated as $\mathrm{AB}$ or $\mathrm{AA}^{\prime}$ or $\mathrm{BB}^{\prime}$ systems respectively. The lower chemical shifts ( $\mathrm{ppm}$ ) obtained for $\mathrm{H} \alpha$ and higher chemical shifts (ppm) obtained for $\mathrm{H} \beta$ in this series of ketones. The vinyl protons give an $\mathrm{AB}$ pattern and the $\beta$-proton doublets were well separated from the signals of the aromatic protons. The assigned vinyl proton chemical shifts $\delta(\mathrm{ppm})$ of all ketones were presented in Table 2. 
In nuclear magnetic resonance spectra, the proton or the ${ }^{13} \mathrm{C}$ chemical shifts $(\delta)$ depends on the electronic environment of the nuclei concerned. The assigned vinyl proton chemical shifts $(\mathrm{ppm})$ have been correlated with reactivity parameters using Hammett equation in the form of

$$
\log \delta=\log \delta_{0}+\rho \sigma
$$

where $\delta_{0}$ is the chemical shift of unsubstitued ketones.

The assigned $\mathrm{H}_{\alpha}$ and $\mathrm{H}_{\beta}$ proton chemical shifts (ppm) are correlated with various Hammett sigma constants. The results of statistical analysis $[1,16,40,41]$ are presented in Table 3. The obtained correlation is satisfactory for $\mathrm{H}_{\alpha}$ with $\mathrm{R}$ parameter $(\mathrm{r}=0.905)$. All Hammett substituent constants, $\mathrm{R}$ parameters were fail in correlation. A fair degree of correlation is obtained for $\mathrm{H}_{\beta}$ proton chemical shifts $(\mathrm{ppm})$ with Hammett sigma $\sigma(\mathrm{r}=0.905), \sigma^{+}(\mathrm{r}=0.906)$, constants and $\mathrm{R}(\mathrm{r}=$ 0.906) parameters. Other Hammett constant $\sigma_{\mathrm{I}}, \sigma_{\mathrm{R}}$ and $\mathrm{F}$ parameter have shown poor correlation. The failure in correlation is due to the reason stated earlier. Application of Swain-Lupton's [44] treatment to the relative chemical shifts of $\mathrm{H}_{\alpha}$ and $\mathrm{H}_{\beta}$ with $\mathrm{F}$ and $\mathrm{R}$ values is successful with resonance, inductive effect generates the multi regression equations 18-21.

$$
\begin{aligned}
& \delta_{\mathrm{H \alpha}}(\mathrm{ppm})=7.436( \pm 0.227)-0.581( \pm 0.461) \sigma_{\mathrm{I}}+0.338( \pm 0.357) \sigma_{\mathrm{R}} \\
& (R=0.953, \mathrm{n}=9, \quad \mathrm{P}>95 \%) \\
& \delta_{\text {На }}(\mathrm{ppm})=7.413( \pm 0.178)-0.535( \pm 0.355) \mathrm{F}+0.205( \pm 0.307) \mathrm{R} \\
& (R=0.960, \quad \mathrm{n}=9, \quad \mathrm{P}>95 \%) \\
& \delta_{\mathrm{H} \beta}(\mathrm{ppm})=8.00( \pm 0.192)+0.219( \pm 0.391) \sigma_{\mathrm{I}}+0.552( \pm 0.303) \sigma_{\mathrm{R}} \\
& (R=0.961, \mathrm{n}=9, \quad \mathrm{P}>95 \%) \\
& \delta_{\mathrm{H} \beta}(\mathrm{ppm})=8.053( \pm 0.145)+0.167( \pm 0.290) \mathrm{F}+0.605( \pm 0.251) \mathrm{R} \\
& (R=0.971, \mathrm{n}=9, \quad \mathrm{P}>95 \%)
\end{aligned}
$$

\section{1. $4{ }^{13} \mathrm{C}$ NMR spectral study}

Organic chemists, physical organic chemists, spectral analysts, and scientists $[1,16,40,41]$ have made extensive study of ${ }^{13} \mathrm{C}$ NMR spectra for a large number of different ketones and styrenes. The assigned vinyl $\mathrm{C}_{\alpha}, \mathrm{C}_{\beta}$ and carbonyl carbon chemical shifts are presented in Table 2. The results of statistical analysis are given in Table 3. The $\mathrm{C}_{\alpha}$ chemical shifts (ppm) gave satisfactory correlation with Hammett $\sigma(\mathrm{r}=0.904)$ and $\sigma^{+}(\mathrm{r}=0.905)$ constants. Remaining Hammett constant, $\mathrm{F}$ and $\mathrm{R}$ parameters gave poor correlation. The chemical shifts $(\mathrm{ppm})$ of $\mathrm{C}_{\beta}$ carbon with Hammett constant $\sigma(r=0.907), \sigma^{+}(r=0.905), \sigma_{I}(r=0.905), \sigma_{R}(r=0.900)$ and $R(r=$ $0.905)$ parameter gave satisfactory correlation. The $F$ parameter was fail in correlation. This is due to reasons stated earlier with the resonance conjugative structure shown in Figure 1. The carbonyl carbon chemical shifts (ppm) of all ketones gave poor correlated with all Hammett $\sigma$ constants, $\mathrm{F}$ and $\mathrm{R}$ parameter due to reason stated earlier and associated with resonance conjugative structure shown in Figure. 2. The Swain Lupton's [44] parameter correlations were satisfactorily obtained within these carbon chemical shifts and the regression equations are given in 22-27.

$$
\begin{aligned}
\delta \mathrm{C} \alpha(\mathrm{ppm})=\quad & 121.196( \pm 1.834)+3.211( \pm 1.728) \sigma_{\mathrm{I}}+2.613( \pm 2.890) \sigma_{\mathrm{R}} \\
& (R=0.945, \mathrm{n}=9, \quad \mathrm{P}>90 \%) \\
\delta \mathrm{C} \alpha(\mathrm{ppm})=\quad & \begin{array}{l}
122.041( \pm 1.488)+1.935( \pm 0.966) \mathrm{F}+3.608( \pm 2.565) \mathrm{R} \\
(R=0.950, \mathrm{n}=9, \quad \mathrm{P}>95 \%)
\end{array}
\end{aligned}
$$




$$
\begin{aligned}
& \delta \mathrm{C} \beta(\mathrm{ppm})=137.769( \pm 2.892)+13.937( \pm 5.880) \sigma_{\mathrm{I}}+12.893( \pm 4.557) \sigma_{\mathrm{R}} \\
& (R=0.983, \quad \mathrm{n}=9, \quad \mathrm{P}>95 \%) \\
& \begin{aligned}
\delta \mathrm{C} \beta(\mathrm{ppm})=\quad & 138.410( \pm 2.650)+12.335( \pm 5.283) \mathrm{F}+12.899( \pm 4.568) \mathrm{R} \\
& (R=0.979, \quad \mathrm{n}=9, \quad \mathrm{P}>95 \%)
\end{aligned} \\
& \delta \mathrm{CO}(\mathrm{ppm})=192.484( \pm 4.942)+0.594( \pm 10.047) \sigma_{\mathrm{I}}+11.043( \pm 7.787) \sigma_{\mathrm{R}} \\
& (R=0.958, \quad \mathrm{n}=9, \quad \mathrm{P}>95 \%) \\
& \delta \mathrm{CO}(\mathrm{ppm})=191.984( \pm 4.403)+0.209( \pm 0.776) \mathrm{F}+7.535( \pm 7.588) \mathrm{R} \ldots \ldots \\
& (R=0.938, \quad \mathrm{n}=9, \quad \mathrm{P}>90 \%)
\end{aligned}
$$

\section{ANTIMICROBIAL ACTIVITIES}

Chalcones possess a wide range of biological activities such as antibacterial [13-15], antifungal [17-19], antiviral [10-12], antifeedant [34], anticancer [20-22], antimalarial [7-9], and antioxidant [29], immunomodulatory [35], activities. These multipronged activities present in different chalcones are examined against respective microbes-bacteria's and fungi.

\section{1. Antibacterial sensitivity assay}

Antibacterial sensitivity assay was performed using Kirby-Bauer [46] disc diffusion technique. In each Petri plate about $0.5 \mathrm{ml}$ of the test bacterial sample was spread uniformly over the Solidified Mueller Hinton agar using sterile glass spreader. Then the discs with $5 \mathrm{~mm}$ diameter made up of Whatmann No.1 filter paper, impregnated with the solution of the compound were placed on the medium using sterile forceps. The plates were incubated for 24 hours at $37{ }^{\circ} \mathrm{C}$ by keeping the plates upside down to prevent the collection of water droplets over the medium. After 24 hours, the plates were visually examined and the diameter values of the zone of inhibition were measured. Triplicate results were recorded by repeating the same procedure.

The antibacterial screening effect of synthesized chalcones is shown in Figure 3. (Plates 110). The zone of inhibition is compared using Table 4 and the Clustered column Chart is shown in Figure 4. A antibacterial activity has been possessed by all the Compounds on the microorganisms in general. All the compounds showed activities except 2- F, 4-F, 2- $\mathrm{OCH}_{3}$ has very good active against Escherichia coli. The substituent $3-\mathrm{Cl}$ and $2-\mathrm{NO}_{2}$ have high activity against $B$. subtilis, $\mathrm{H}$, 4-Cl, 2-F has a maximum activity against $P$. aeruginosa expect other substituent. 
Table 4. The antibacterial activities of 2'-hydroxyphenylchalcones.

\begin{tabular}{|c|c|c|c|c|c|c|}
\hline \multirow{3}{*}{ S. No. } & \multirow{3}{*}{$\mathbf{X}$} & \multicolumn{5}{|c|}{ Zone of Inhibition (mm) } \\
\hline & & \multicolumn{3}{|c|}{ Gram positive Bacteria } & \multicolumn{2}{|c|}{ Gram negative Bacteria } \\
\hline & & B. subtilis & M. luteus & S. aureus & E. Coli & P. aeruginosa \\
\hline 1 & $\mathrm{H}$ & 8 & 7 & 7 & 7 & 9 \\
\hline 2 & $2-\mathrm{Br}$ & 7 & - & 7 & 8 & - \\
\hline 3 & $3-\mathrm{Cl}$ & 13 & 7 & 8 & 8 & 7 \\
\hline 4 & $4-\mathrm{Cl}$ & 7 & 7 & 7 & 7 & 10 \\
\hline 5 & $2-\mathrm{F}$ & - & - & 7 & - & 8 \\
\hline 6 & $4-\mathrm{F}$ & - & 6 & - & - & - \\
\hline 7 & $2-\mathrm{OCH}_{3}$ & - & - & - & - & 7 \\
\hline 8 & $4-\mathrm{OCH}_{3}$ & 7 & - & - & 7 & - \\
\hline \multirow[t]{3}{*}{9} & $2-\mathrm{NO}_{2}$ & 12 & 8 & 8 & 9 & 6 \\
\hline & Ampicillin & 14 & 15 & 16 & 15 & 14 \\
\hline & DMSO & - & - & - & - & - \\
\hline
\end{tabular}

\section{2. Antifungal sensitivity assay}

Antifungal sensitivity assay was performed using Kirby-Bauer [46] disc diffusion technique. PDA medium was prepared and sterilized as above. It was poured (ear bearing heating condition) in the Petri-plate which was already filled with $1 \mathrm{ml}$ of the fungal species. The plate was rotated clockwise and counter clock-wise for uniform spreading of the species. The discs were impregnated with the test solution.

The test solution was prepared by dissolving $15 \mathrm{mg}$ of the chalcone in $1 \mathrm{ml}$ of DMSO solvent. The medium was allowed to solidify and kept for 24 hours. Then the plates were visually examined and the diameter values of zone of inhibition were measured. Triplicate results were recorded by repeating the same procedure. 


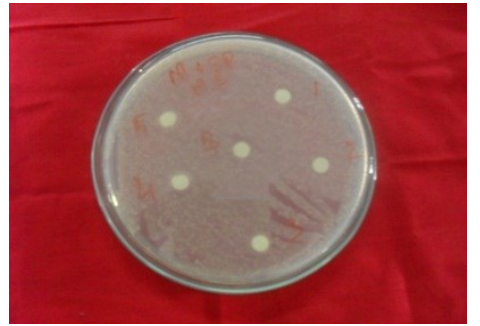

PLATE-1

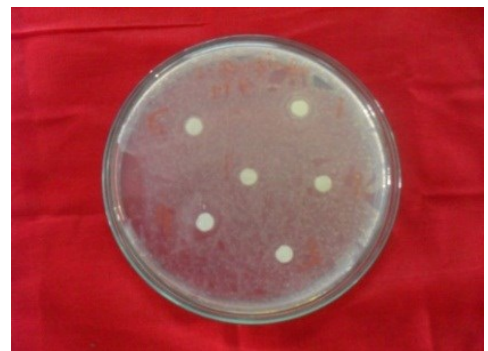

PLATE-3

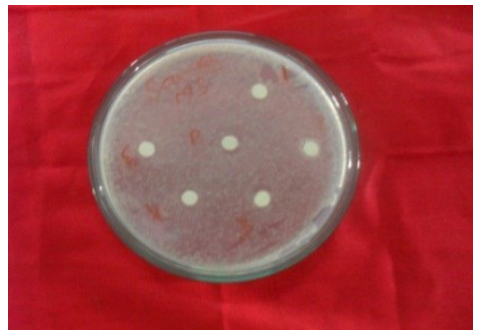

PLATE-5

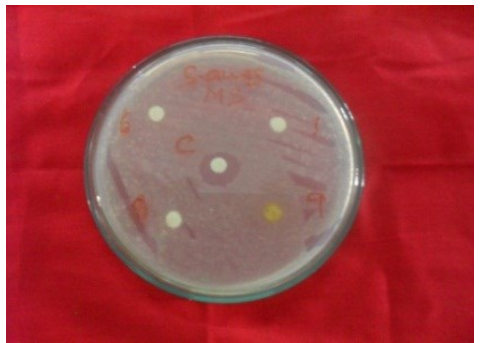

PLATE-7

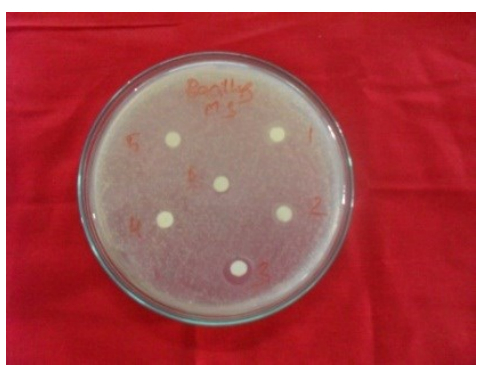

PLATE-9

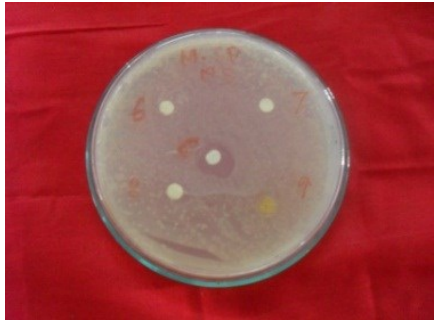

PLATE-2

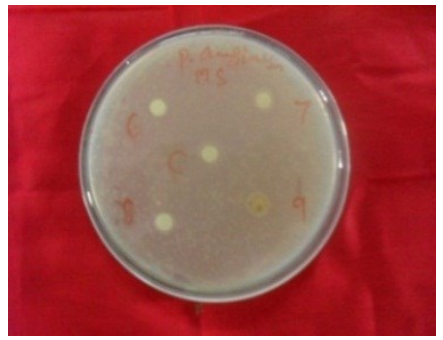

PLATE-4

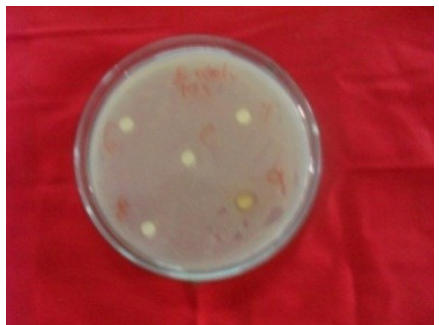

PLATE-6

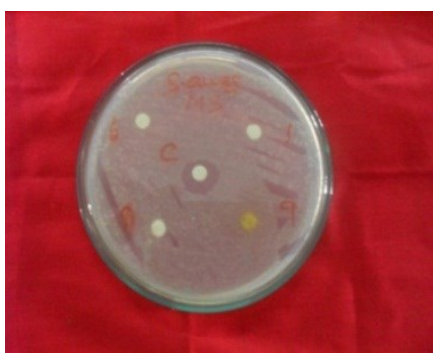

PLATE-8

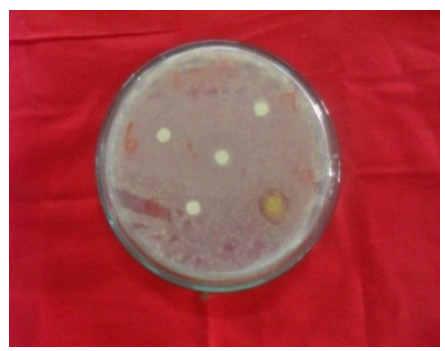

PLATE-10

Fig. 3. Antibacterial activity of substituted 2-hydroxyphenylketone - petri dishes. 


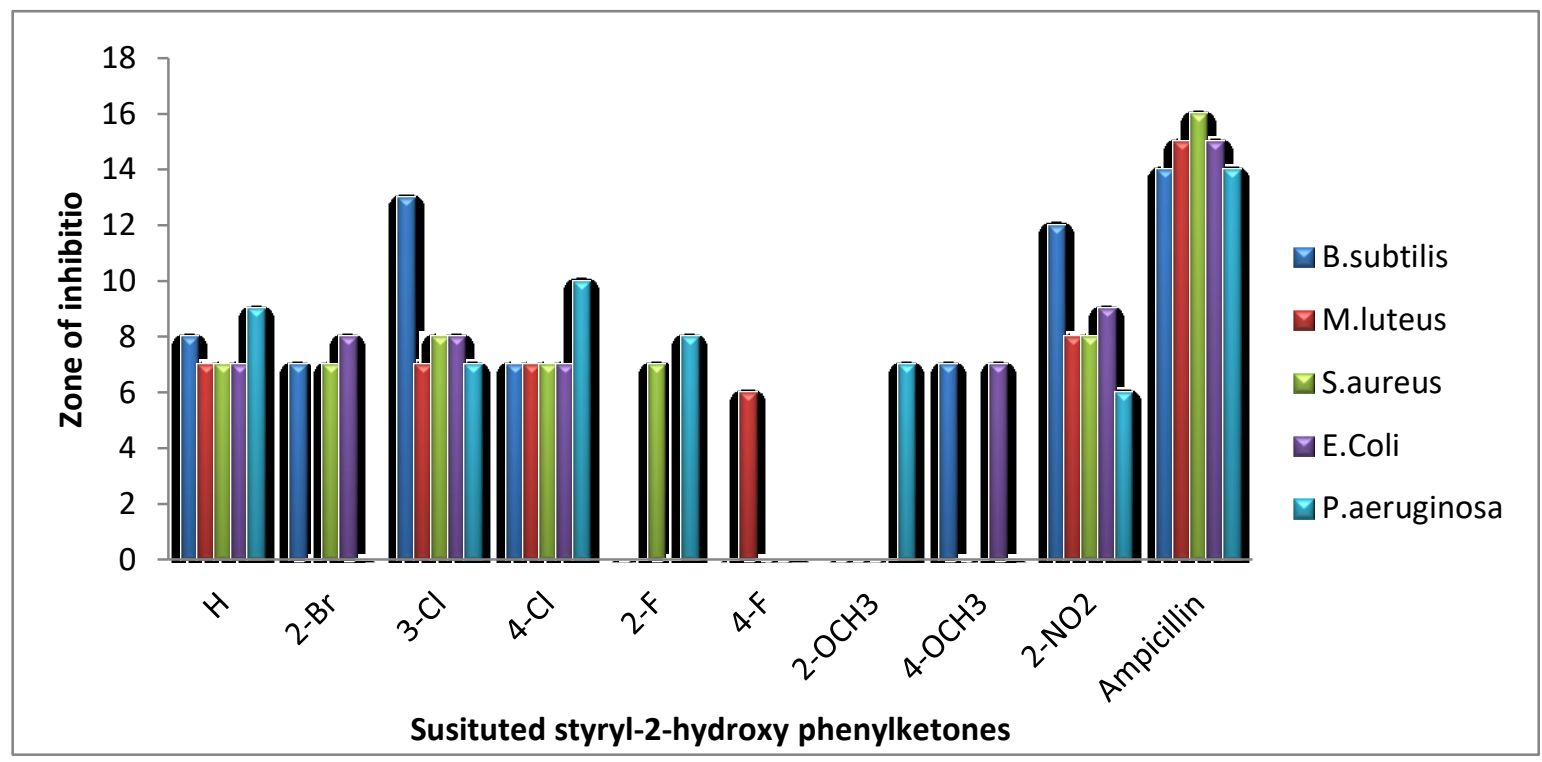

Fig. 4. Antibacterial activity of substituted 2-hydroxyphenyl-styrylketone - clustered column chart.

Table 5. Anti-fungal activity of substituted 2-hydoxyphenyl-styrylketone.

\begin{tabular}{|c|c|c|c|}
\hline \multirow{2}{*}{ S. No. } & \multirow{2}{*}{$\mathbf{X}$} & \multicolumn{2}{|c|}{ Zone of Inhibition (mm) } \\
\cline { 2 - 4 } & & A. niger & T. Viride \\
\hline 1 & $\mathrm{H}$ & - & 6 \\
\hline 2 & $2-\mathrm{Br}$ & - & 6 \\
\hline 3 & $3-\mathrm{Cl}$ & 8 & - \\
\hline 4 & $4-\mathrm{Cl}$ & 9 & 9 \\
\hline 5 & $2-\mathrm{F}$ & - & 8 \\
\hline 6 & $4-\mathrm{F}$ & 9 & 7 \\
\hline 7 & $2-\mathrm{OCH}_{3}$ & - & 7 \\
\hline 8 & $4-\mathrm{OCH}_{3}$ & 8 & 6 \\
\hline 9 & $2-\mathrm{NO}_{2}$ & - & 14 \\
\hline & Miconazole & 13 & - \\
\hline & DMSO & - & - \\
\hline
\end{tabular}

The antifungal activities of substituted chalcones synthesized in the present study are shown in Figure 5 for Plates (1-4) and the zone of inhibition values of the effect is given in Table 5. The clustered column chart, shown in Figure 6, reveals that all the compounds have excellent antifungal 
activity against all the two fungal species namely $A$. niger, and $T$. Viridi The Chalcones with 4-F, 4$\mathrm{Cl}$, and $4-\mathrm{OCH}_{3}$. substituents have shown greater antifungal activity against. T. Viridi and A. niger.

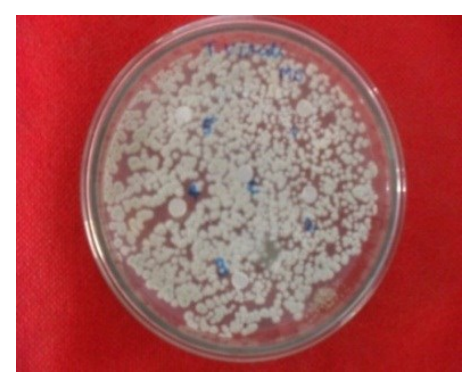

Plate-1

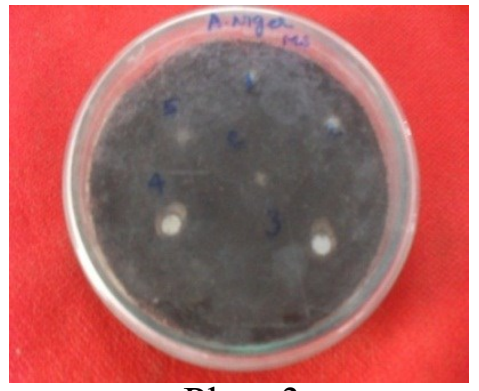

Plate-3

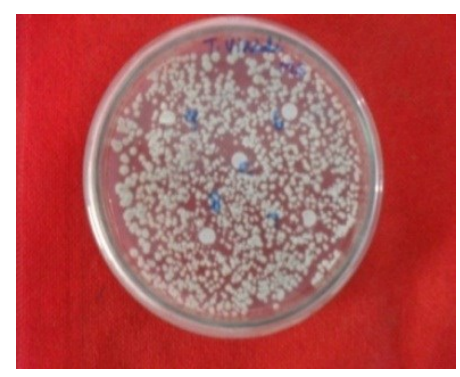

Plate-2

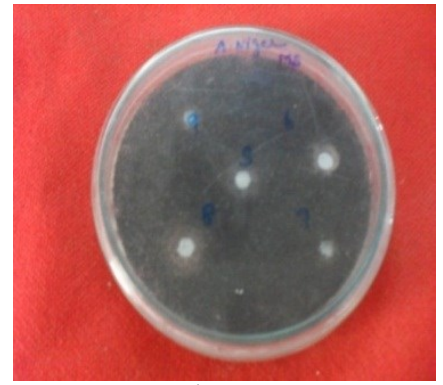

Plate-4

Fig. 5. Antifungal activity of substituted 2-hydroxyphenyl-styrylketone - petri dishes.

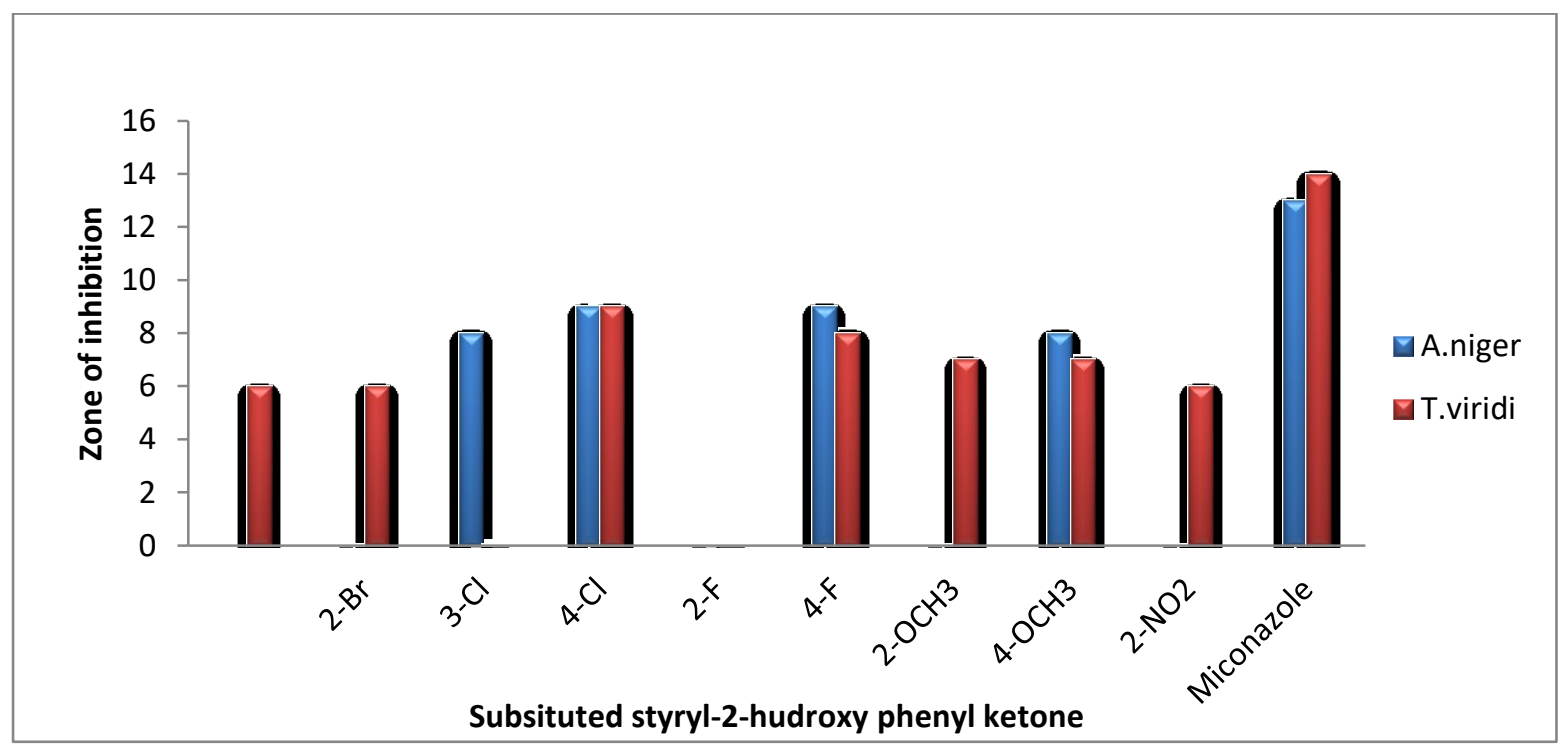

Fig. 6. Antifungal activity of substituted 2-hydroxyphenyl-styrylketone - clustered column chart.

\section{CONCLUSION}

Some chalcones have been synthesized by condensation of 2-hydroxyacetophenone and substituted benzaldehydes using microwave irradiation in the presence of $\mathrm{SiO}_{2}-\mathrm{H}_{3} \mathrm{PO}_{4}$ under solvent free conditions. The chalcones have been characterized by their physical constants, spectral data. The UV, IR, NMR spectral data of these chalcones has been correlated with Hammett substituent constants, $\mathrm{F}$ and $\mathrm{R}$ parameters. From the results of statistical analyses the effects of substituent on the spectral data have been studied. The antimicrobial activities of all synthesized chalcone have been studied using Bauer-Kirby method. 


\section{Acknowledgement}

The authors thank DST NMR facility, Department of Chemistry, Annamalai University, Annamalainagar - 608 002, for recording NMR spectra of all compounds.

\section{References}

[1] Ranganathan R., Arulkumaran R., Kamalakkannan D., Vanangamudi G., Thirunarayanan G., IUP J. Chem 4(2) (2011) 60-70.

[2] Sritularak K., Likhitwayawuid D., Phytochem. 67 (2006) 812-817.

[3] Nowakowska Z. A., Eur. J. Med. Chem. 42 (2007) 125-137.

[4] Narender T., Shweta Tanvir K., Srinivasa Rao M., Srivastava K., Puri S. K., Bioorg. Med. Chem. Lett. 15 (2005) 2453-2455.

[5] Zhang Y, Shi S,. Zhao M,. Jiang Y., Tu P., Biochem. Syst. Ecol. 34 (2006) 766-69.

[6] Anjani Solankee, Ghanshyam Patel, Sejal Solankee, R. J. Chem 1 (2008) 591-595.

[7] Dominguez J. N., Charris J. E., Lobo G., de Dominguez N. G., Moreno M. M., Riggione F., Sanchez E., Olsen J., Rosenthal P. J., Eur. J. Med. Chem 36 (2001) 555-560.

[8] Li R., Kenyon G. L., Cohen F. E., Chen X., Gong B., Dominguez J. N., Davidson E., Kurzban G., Miller R. E., Nuzum E. O., Rosenthal P. J., Mckerrow J. H., J. Med. Chem 38 (1995) 5031-5037.

[9] Liu M., Wilairat P., Go M. L., J. Med. Chem 44 (2001) 4443-4452.

[10] Cheenpracha S., Karapai C., Ponglimanont C., Subhadhirasakul S., Tewtrakal S., Bioorg. Med. Chem. 14 (2006) 1710-1715.

[11] Wu J. H., Wang X. H., Yi Y. H., Lee K. H., Bioorg. Med. Chem. Lett. 13 (2003) 1813-1815.

[12] Onyilagna J. C., Malhotra B., Elder M., Towers G. H. N., Can. J. Plant Pathol. 19 (1997) 133-137.

[13] Jae In Lee, Hwa Soo Son, Hyun Park., Bull. Korean Chem. Soc. 25 (2004) 1945-1947.

[14] Avila H. P., de Fatima E., Smania A., Monache F. D., Junior A. S., Bioorg. Med. Chem. 16 (2008) 9790-9794.

[15] Nielsen S. F., Larsen M., Bosen T., Schonning K., Kromann H., J. Med. Chem. 48 (2005) 2667-2677.

[16] Arulkumaran R., Sundararajan R., Vanangamudi G., Subramanian M., Ravi K., Sathiyendidran V., Srinivasan S., Thirunarayanan G., IUP J. Chem. 3 (2010) 82-98.

[17] Lahtchev K. L., Batovska D. I., Parushev St. P., Ubiyvovk V. M., Sibirny A. A., Eur. J. Med. Chem. 43 (2008) 2220-2228.

[18] Lawrence N. J., Patterson R. P., Ooi L. L., Cook D., Ducki S., Bioorg. Med. Chem. Lett. 16 (2006) 5844-5848.

[19] Batovska D., Parushev St., Slavova A., Bankova V., Tsvetkova I., Ninova M., Najdenski H., Eur. J. Med. Chem. 42 (2007) 87-92.

[20] Modzelewska A., Pettit C., Achanta G., Davidson N. E., Huang P., Khan S. R., Bioorg. Med. Chem 14 (2006) 3491-3495.

[21] Chen F. C., Bioorg. Med. Chem. 10 (2002) 2795-2802. 
[22] Kumar S. K., Hager E., Pettit C., Gurulingappa H., Davidson N. E., Khan S. R., J. Med. Chem. 46 (2003) 2813-2815.

[23] Suryawanshi S. N., Chandra N., Kumar P., Porwal J., Gupta S., Eur. J. Med. Chem. 43 (2008) 2473-2478.

[24] Won S. J., Liu C. T., Tsao L. T., Weng J. R., Ko H. H., Wang J. P., Lin C. N., Eur. J. Med. Chem. 40 (2005) 103-112.

[25] De Leon E. J., Alcaraz M. J., Dominguez J. N., Charris J., Terencio M. C., Inflamm. Res. 52 (2003) 246-257.

[26] Viana G. S., Bandeira M. A., Matos F. J., Phytomedicine. 10 (2003) 189-195.

[27] Murakami S., Muramatsu M., Aihara H., Otomo S., Biochem. Pharmacol. 42 (1991) 14471451.

[28] Satyanarayana M., Tiwari P., Tripathi B. K., Srivastava A. K., Pratap R., Bioorg. Med. Chem. 12 (2004) 883-889.

[29] Dimmock J. R., Elias D. W., Beazely M. A., Kandepu N. M., Curr. Med. Chem 6 (1999) 1125-1149.

[30] Mukherjee S., Kumar V., Prasad A. K., Raj H. G., Bracke M. E., Olson C. E., Jain S. C., Parmar V. S., Bioorg. Med. Chem. 9 (2001) 337-347.

[31] Zhao L. M., Jin H. S., Sun L. P., Piao H. R., Quan Z. S., Bioorg. Med. Chem. Lett. 15 (2005) 5027-5029.

[32] Thirunarayanan G., J. Indian Chem. Soc 84 (2008) 447-451.

[33] Wood J. E., Munro M. H. G., Blunt J. W., Perry N. B., Walker J. R. C., Ward J., N. Z., J. Bot. 37 (1999) 167-169.

[34] (a) Thirunarayanan G., J. Indian Chem. Soc. 84 (2008) 447-451.

(b) Thirunarayanan G., Surya S., Srinivasan S., Vanangamudi G., Sathyendiran V., Spectrochim Acta. 75A (2010) 152-156.

[35] Liu X. L., Xu Y. J., Go M. L., Eur. J. Med. Chem. 43 (2008) 1681-1687.

[36] Avila H. P., de Fatima E., Smania A., Monache F. D., Junior A. S., Bioorg. Med. Chem. 16 (2008) 9790-9794.

[37] Nielsen S. F., Larsen M., Bosen T., Schonning K., Kromann H., J. Med. Chem. 48 (2005) 266-777.

[38] Lin Y. M., Zhou Y., Flavin M. T., Zhou L. M., Nie W., Chen F. C., Bioorg. Med. Chem. 10 (2002) 2795-2802.

[39] Lahtchev K. L., Batovska D. I., St. Parushev P., Ubiyvovk V. M., Sibirny A. A., Eur. J. Med. Chem. 43 (2008) 2220-2228.

[40] Subramanian M., Vanangamudi G., Thirunaryanan G., Spectrochim. Acta. 2013. DOI:10.1016/j.saa.2013.03.023.

[41] Janaki P., Sekar K. G., Thirunarayanan G., Organic Chem: An Indian J. 9 (2013) 68-80.

[42] Srivatsava Y. K., Rasayan. J. Chem. 1(4) (2008) 884-886.

[43] Sing S., Sharma P. K., Kumar N., Dudhe R., J. Adv. Sci. Res. 2 (2011) 37.

[44] Swain C. G., Lupton E C. Jr., J. Am. Chem. Soc 90 (1968) 4328-4337.

[45] Hays W. P., Timmons C. J., Spectrochem. Acta 24A (1968) 323-334. 
[46] Bauer A. W., Kirby W. M. M., Sherris J. C., Truck M., Am. J. Clin. Pathol 45 (1996) 493498.

[47] R. Arulkumaran, S. Vijayakumar, R. Sundararajan, S. P. Sakthinathan, D. Kamalakkannan, R. Suresh, K. Ranganathan, G. Vanangamudi, G. Thirunarayanan, International Letters of Chemistry, Physics and Astronomy 4 (2012) 17-38.

[48] K. Ranganathan, R. Suresh, D. Kamalakkannan, R. Arulkumaran, R. Sundararajan, S. P. Sakthinathan, S. Vijayakumar, G. Vanangamudi, K. Thirumurthy, P. Mayavel, G.

Thirunarayanan, International Letters of Chemistry, Physics and Astronomy 4 (2012) 66-75.

[49] R. Arulkumaran, S. Vijayakumar, R. Sundararajan, S. P. Sakthinathan, D. Kamalakkannan, R. Suresh, K. Ranganathan, P. R. Rajakumar, G. Vanangamudi, G. Thirunarayanan, International Letters of Chemistry, Physics and Astronomy 5 (2013) 21-38. 